\title{
Planar liquid crystal polarization optics for augmented reality and virtual reality: from fundamentals to applications
}

\author{
Jianghao Xiong and Shin-Tson Wu*
}

\begin{abstract}
Planar and ultrathin liquid crystal (LC) polarization optical elements have found promising applications in augmented reality (AR), virtual reality (VR), and photonic devices. In this paper, we give a comprehensive review on the operation principles, device fabrication, and performance of these optical elements. Optical simulations methods for optimizing the device performance are discussed in detail. Finally, some potential applications of these devices in AR and VR systems are illustrated and analyzed.
\end{abstract}

Keywords: Liquid crystal, Polarization holography, Flat optics, Virtual reality, Augmented reality

\section{Introduction}

Augmented reality (AR) and virtual reality (VR) are promising candidates for next-generation mobile platform. They have potential to revolutionize the ways we perceive and interact with various digital information [1-4]. Their widespread applications cover education, engineering, entertainment, and healthcare, just to name a few. Unlike traditional flat panel displays, AR and VR embrace the concept of 3D digital image and promote a deeper level of human-computer interactions. The major difference between AR and VR is that AR superimposes digital contents with real environment so that it remains see-through capability, while VR is completely immersive. However, to vividly display $3 \mathrm{D}$ contents imposes unprecedented technical challenges from optics viewpoint. Human eye has excellent optical performances like wide field-of-view (FoV) [5], high dynamic range [6, 7], high visual acuity [8], adaptive focus [9], and 3D perception [10]. To build an AR/VR display system satisfying all the above-mentioned requirements is extremely challenging using traditional optics, not to mention the

*Correspondence: swu@creol.ucf.edu

College of Optics and Photonics, University of Central Florida, Orlando, FL 32816 , USA display itself should also have a compact form factor and lightweight for comfortable wearing experience.

After five decades of extensive material research and development, device innovations, and heavy investment on advanced manufacturing technologies, thin-film transistor (TFT) liquid crystal display (LCD) has reached a matured stage. Its widespread applications cover from smartphones, pads, notebook and desktop computers, TVs, to projectors, just to name a few. Recently, mini-LED backlit quantum-dot LCD offers a comparable dynamic range, grayscale bit depth, and color gamut with its organic light-emitting diode (OLED) counterpart $[11,12]$. Besides displays, LCs exhibit some other attractive properties, such as phase-only modulation and photo-patternable characteristic. These properties can be used to dynamically manipulate the wavefront of a light source and create new photonic devices, such as grating and lens. Lately, LC-based diffractive optics [13-16] has attracted increasing interest due to its advantages in high efficiency, polarization selectivity, switching ability, and ultrathin form factor. The ultrathin LC optical elements, with thickness of several micrometers, enable very compact and lightweight optical components with electronic modulation capacity compared to their 
counterparts using mechanical modulation. The fastswitching and precise wavefront control further distinguish these LC optical elements. These unique properties can be employed to address the major challenges in AR/ VR displays and, in the meantime, enable novel photonic applications.

In this review, we will begin with a brief introduction to the fundamental LC physics, which is necessitated to understand the operation principles of the devices, and then give some examples of practical applications. The LC physics serves as the foundation for the LC devices described here. The optical properties of these devices offer further insights into their potential functionalities. Finally, some exemplary applications of these planar, ultrathin LC optical elements illustrate how their unique properties help solving current issues of neareye displays. This review aims to give a comprehensive understanding of planar LC optics and inspire deeper investigations into the near-eye displays and other promising photonic devices.

\section{Formation of planar LC optical elements}

\subsection{Basics of liquid crystal physics}

A thermotropic LC exists between solid and isotropic phases. An anisotropic LC is more ordered than an isotropic liquid and exhibits a certain degree of orientational order. On the other hand, it is less rigid than a crystalline solid and can flow easily. Several kinds of thermotropic LCs have been discovered, including nematic, smectic, and columnar (or discotic) phases [17]. The order of these phases increases accordingly. Nematic phase has no positional order and it acts like a 3D liquid. The LC molecules can move freely in 3D space, as shown in Fig. 1a. Smectic phase has 1D positional order, which resembles stacked 2D liquid layers, as Fig. 1b depicts. On the other hand, columnar phase has 2D positional order and behaves like an array of 1D liquid tubes, as sketched in Fig. 1c. Due to mechanical instability and defects, smectic and columnar phases are less commonly used in practical applications than nematic.

The presently widely used flat panel displays use nematic LCs. If we dope a chiral compound into a nematic host, the LC will form a helical structure depicted in Fig. 1d. This helical structure is called cholesteric liquid crystal (CLC). Therefore, we can regard CLC as a derivative of nematic. Meanwhile, there is another phase existing in between cholesteric phase and isotropic phase, called blue phase [18]. But the operating voltage of blue phase is quite high so that its application is still limited. Thus, we will not discuss it here. Detailed descriptions of LC phases and their physical properties can be found in [17]. The planar optics we present here mainly involves nematic and CLC phases.

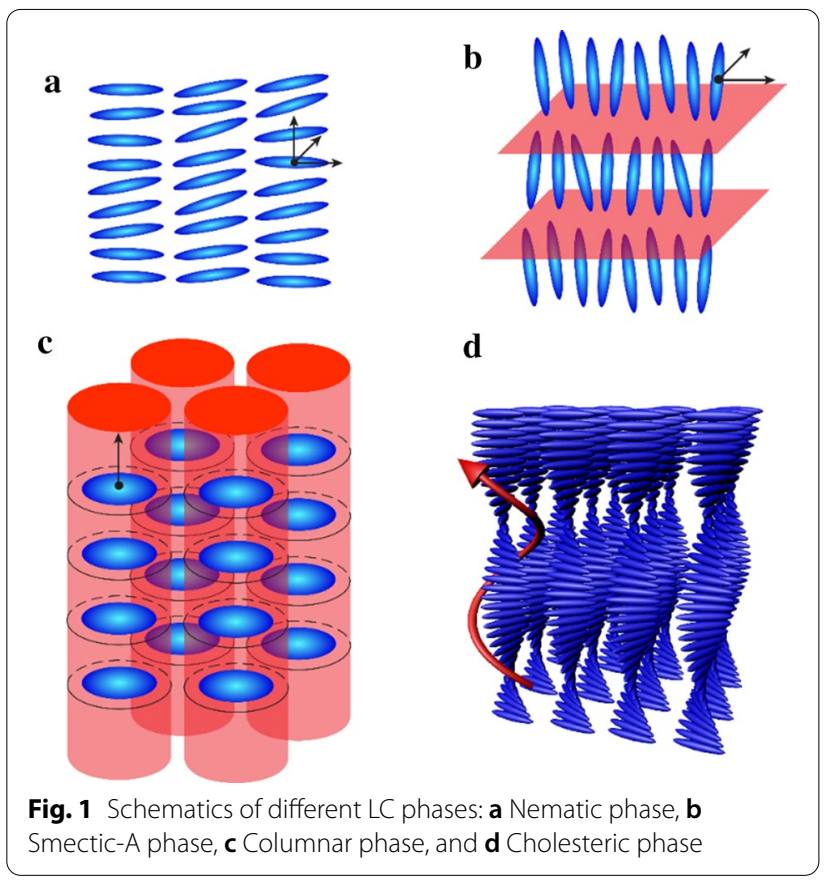

A nematic LC usually consists of elongated rod-like molecules. Thus, we can use a unit vector $\widehat{\boldsymbol{n}}$ called the LC director to describe the averaged orientation of local molecules. As shown in Fig. 2a, the LC director $\widehat{\boldsymbol{n}}$ can be spatially variant. If we look at a local region, the molecular orientations are distributed near director $\widehat{\boldsymbol{n}}$. Therefore, we can define a scalar order parameter $S$ as

$$
S=\frac{1}{2}\left\langle 3 \cos ^{2} \theta-1\right\rangle,
$$

where \langle\rangle denotes the statistical average and $\theta$ is the angle between the molecules and the director $\widehat{\boldsymbol{n}}$. As the temperature increases, LC undergoes phase transition to an isotropic state. Under such condition, the LC directors are randomly distributed so that $\mathrm{S}=0$. When the temperature is below the melting point, the LC directors are frozen to a crystalline state, and $S=1$. In a nematic state, $S$ is usually in the 0.6 to 0.7 range, depending on the temperature. The most generalized description of LC behavior uses a tensor order parameter $\boldsymbol{Q}$ defined as

$$
Q_{j i}=\frac{S}{2}\left(3 n_{i} n_{j}-\delta_{i j}\right),
$$

where $\delta_{i j}$ denotes Kronecker delta. Because Q contains information of both order parameter and director, it can describe the thermotropic phase transition and LC dynamics. But in most applications at a given temperature, the order parameter is usually a constant, thus a simpler way to describe the LC dynamic behaviors is to only use the director $\widehat{\boldsymbol{n}}$. 

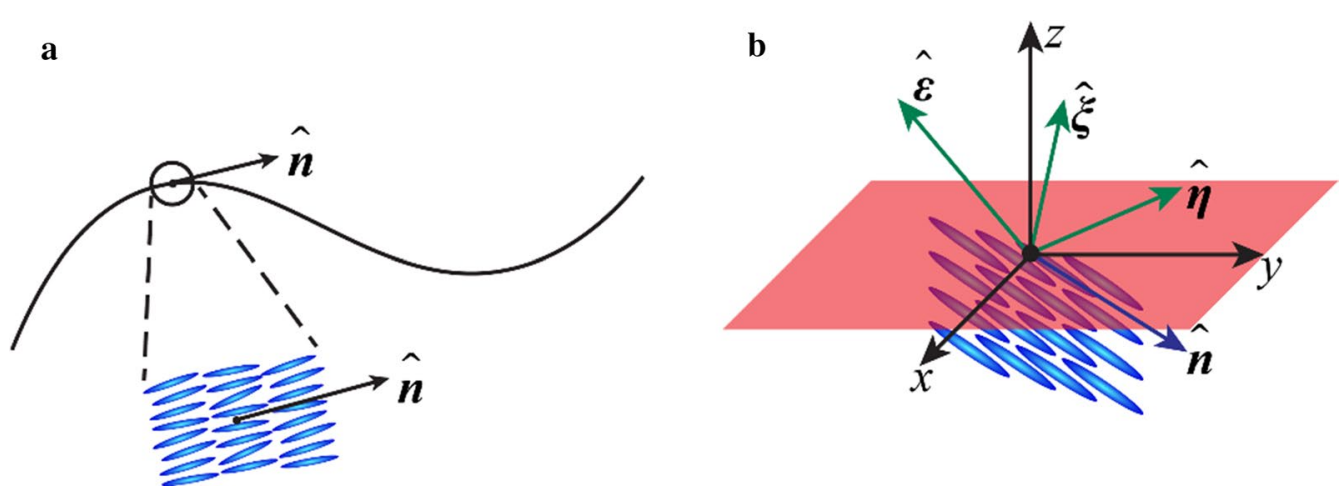

Fig. 2 a Schematics of local LC director. b Diagram of local coordinate system corresponding to the diagonalized anchoring tensor

To acquire spatial and temporal information of the LC director $\widehat{\boldsymbol{n}}(x, t)$, free energy model is used. The total free energy $F_{\text {total }}$ consists of three parts: the elastic energy $F_{e l}$ from director reorientations, the surface free energy $F_{s}$ from interaction between LC and surface anchoring, and the electromagnetic field free energy $F_{\text {field }}$ from dipole coupling between LC and electric/magnetic fields.

According to Frank-Oseen model [17], the elastic free energy density $f_{e l}$ can be expressed as

$$
\begin{aligned}
f_{e l}= & \frac{1}{2} K_{1}(\nabla \cdot \widehat{\boldsymbol{n}})^{2}+\frac{1}{2} K_{2}\left(\widehat{\boldsymbol{n}} \cdot \nabla \times \widehat{\boldsymbol{n}}+\frac{2 \pi}{P}\right)^{2} \\
& +\frac{1}{2} K_{3}|\widehat{\boldsymbol{n}} \times \nabla \times \widehat{\boldsymbol{n}}|^{2},
\end{aligned}
$$

where $K_{1}, K_{2}$ and $K_{3}$ is the splay, twist, and bend elastic constant, respectively, and $P$ is the helical pitch of CLC with the director reorienting from 0 to $2 \pi$. When there is no chiral dopant, $P$ is infinity. From Eq. (3), we can see that in order for $f_{e l}$ to be zero, all the spatial derivatives of $\widehat{\boldsymbol{n}}$ should be zero, which indicates $\widehat{\boldsymbol{n}}$ should be a constant across the space. When $P$ is finite, it is easy to verify that $\widehat{\boldsymbol{n}}=\left(\cos \frac{2 \pi}{P} z, \sin \frac{2 \pi}{P} z, 0\right)$ gives zero $f_{e l}$, which corresponds to a helical structure with pitch length $P$ and the helix is along the $z$ axis. Also, because the value of $f_{e l}$ is not dependent on the choice of coordinates, any rotation of the coordinate system should produce the same result, which means a helical structure with period $P$ and arbitrary helical axis orientation leads to $f_{e l}=0$.

When an LC is subject to an external field, the free energy density arising from LC's electric and magnetic anisotropies can be expressed as:

$$
f_{\text {field }}=-\frac{1}{2} \Delta \varepsilon(\widehat{\boldsymbol{n}} \cdot \boldsymbol{E})^{2}-\frac{1}{2} \Delta \chi(\widehat{\boldsymbol{n}} \cdot \boldsymbol{H})^{2},
$$

where $\boldsymbol{E}$ and $\boldsymbol{H}$ are the electric and magnetic fields, $\Delta \varepsilon$ and $\Delta \chi$ are dielectric and diamagnetic anisotropies.
Depending on the sign of $\Delta \varepsilon$ and $\Delta \chi$, the LC directors could be reoriented to be parallel or perpendicular to the electric field or the magnetic field.

The surface free energy density $f_{s}$ determines how the LC directors align at the interface. The most general form of $f_{s}$ adopts the following $[19,20]$ :

$$
f_{s}=-\frac{1}{2} \sum_{i, j} W_{i j} n_{i} n_{j}
$$

where $W_{i j}$ is the traceless symmetrical anchoring tensor. We can always diagonalize $W_{i j}$ in a local coordinate system $\widehat{\xi}, \widehat{\eta}, \widehat{\boldsymbol{\varepsilon}}$, as shown in Fig. 2b. Also, note that $\widehat{\boldsymbol{n}}$ is a unit vector, thus $f_{s}$ can be rewritten as:

$$
f_{s}=W_{\xi}(\widehat{\boldsymbol{n}} \cdot \widehat{\boldsymbol{\xi}})^{2}+W_{\eta}(\widehat{\boldsymbol{n}} \cdot \widehat{\boldsymbol{\eta}})^{2} .
$$

Here, we ignore the constant term. When $\widehat{\xi}=\widehat{z}$ and $W_{\eta}=0, f_{s}=W_{z}(\widehat{\boldsymbol{n}} \cdot \widehat{\boldsymbol{z}})^{2}$. If $W_{z}<0$, the LC directors tend to align along surface normal $\widehat{z}$. If $W_{z}>0$, LC directors tend to align in $x-y$ plane, which corresponds to degenerate planar anchoring. When $W_{\eta} \neq 0, \widehat{\xi}=\widehat{z}$ and $W_{z}>0$, it corresponds to homogeneous anchoring. Because in this case $\widehat{\eta}$ and $\widehat{\boldsymbol{\varepsilon}}$ have to be in $x-y$ plane, the LC directors tend to align parallel to $\widehat{\eta}$ if $W_{\eta}<0$, or parallel to $\widehat{\varepsilon}$ if $W_{\eta}>0$.

The surface free energy can be viewed as a mechanism to align surface LC with treatment of surface by mechanical rubbing, photoalignment or other techniques. The field free energy offers a way to align bulk LC by applying electric/magnetic fields. The final distribution of bulk LC directors is determined by minimizing the following combined free energy:

$$
F_{\text {total }}=\int_{v}\left(f_{\text {el }}+f_{\text {field }}\right) d v+\int_{s} f_{s} d s .
$$




\subsection{Formation mechanism}

In order to manipulate the LC director configuration, from our above analysis, we can either apply an electric field or by patterning the surface molecular alignment. In practical applications, locally addressable electric field can be achieved by the patterned electrodes. Examples include LC lenses [21, 22] and beam steering devices [2326]. However, to achieve a large-aperture LC lens with high optical quality, the wavefront modulation should be precise, meaning more and finer electrodes are required to increase the degree of modulation freedom. This in turn causes great difficulty in electrode fabrication and device driving. For LC beam steering devices, the diffraction angle is governed by the electrode density and is usually small. For a simple estimate, a liquid-crystalon-silicon device with $3-\mu \mathrm{m}$ pixel pitch would produce a maximum diffraction angle of only $10^{\circ}$ at $\lambda=550 \mathrm{~nm}$. Furthermore, as the diffraction angle increases, the optical efficiency declines rapidly.

On the other hand, locally patterned surface alignment has advantage in precise wavefront modulation. Several patterning methods have been developed, such as micro-rubbing [27, 28], nanoimprinting [29, 30], and photoalignment [31-36]. Among them, photoalignment has the best recording precision and considerably easier fabrication process. The photoalignment technique generally refers to inducing optical anisotropy with the exposure of a polarized light. Among several types of photoalignment approaches are two main methods using photochemical reaction and photoisomerization [32]. The method adopting photochemical reaction like photodimerization enables adjusting LC-surface tilt angle and is essential for applications like multi-domain vertical alignment LCDs [33]. The photoisomerization approach involves a type of polarization-sensitive molecules called azo compounds, where the azobenzene group can transit between low-energy trans state and high-energy cis state, as Fig. 3a depicts. After exposure with an elliptically polarized light, the azo compounds repeatedly undergo trans-cis photoisomerization cycles and gradually rotate to the direction parallel to the short axis of elliptically polarized light, where the absorption is minimum. In the early development stage, azo-dye doped LCs are used to record the wavefront of an interfered polarized light [37-39]. The movement of azo compounds changes the alignment of surrounding LC materials, causing the<smiles>c1ccc(N=Nc2cccc(N=Nc3ccccc3)c2)cc1</smiles>

b<smiles></smiles><smiles>C=CC(=O)OCCCOc1ccc(C(=O)Oc2ccc(OC(=O)c3ccc(OCCOC(=O)C=C)cc3)c(C)c2)cc1</smiles>

d

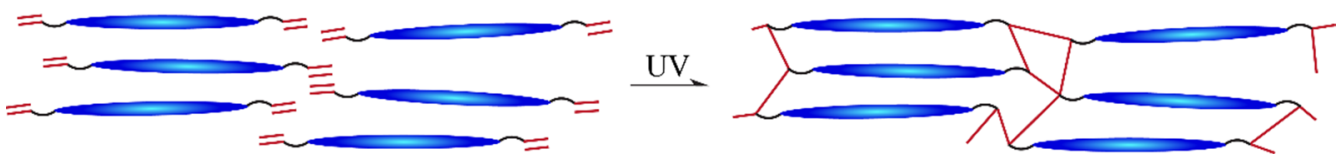

Fig. 3 a Schematics of how azobenzene transits between trans and cis states. Chemical structures of $\mathbf{b}$ brilliant yellow azo-dye and $\mathbf{c}$ reactive mesogen RM257. d Polymerization of reactive mesogen under UV exposure 
LC to form holograms. But the formed holograms usually disappear after the recording beams being removed, due to the reorientation of LC directors. Later, researchers changed the fluidic LC material to LC polymers [34, 40-43]. The recorded holograms remain stable after exposure, but the required dosage increases dramatically. The formation mechanism of such a volume hologram is also complicated, involving a lot of nonlinear interactions $[44,45]$. Afterwards, instead of using a thick film, a thin photoalignment layer is used to align bulk LC. The photoalignment layer can be a photopolymer [34, $35,46]$, an azo-polymer [47], an azo-dye doped polyimide [48-50], or just an azo-dye [14, 51-55]. The azodye alignment layer generally produces a higher optical performance because of its better freedom of molecular movement. Such method is usually called photoalignment polarization holography (PAPH). It has advantages of low exposure dosage, high diffraction efficiency, negligible scattering, and electric-driving potential. Several types of azo-dyes have been used for photoalignment, like methyl red, brilliant yellow, SD-1 and so forth. The chemical structure of a typical azo-dye-based brilliant yellow is shown in Fig. 3b. The LC material used in PAPH could be a fluidic $\mathrm{LC}$ or a reactive mesogen. The latter is basically a polymerizable LC with reactive end groups [56]. The chemical structure of a typical reactive mesogen RM257 is depicted in Fig. 3c. Usually a reactive mesogen and additions like photo-initiator are dissolved in an organic compound. The solution is coated on a substrate.
After the solvent evaporates, the reactive mesogen is in liquid crystalline state. Under ultraviolet (UV) light illumination, the reactive mesogen molecules form the polymer network with high thermal and chemical stabilities, as sketched in Fig. 3d.

To understand the working principle of $\mathrm{PAPH}$, we shall firstly describe how patterns are formed in the photoalignment layer with exposure of a polarized light. From above description of how azo compounds respond to an elliptically polarized light, it is natural to think that linear polarization is a preferred choice for photoalignment, because of its highest ratio between the parallel and perpendicular electric field components. To produce a patterned linear-polarization pattern, one approach is to interfere two circularly polarized beams. As shown in Fig. 4a, when two beams with left-handed circular polarization (LCP) and right-handed circular polarization (RCP) interfere, the electric field can be calculated as:

$$
\left[\begin{array}{l}
1 \\
i
\end{array}\right] e^{-i k_{0} \sin \theta \cdot x}+\left[\begin{array}{c}
1 \\
-i
\end{array}\right] e^{i k_{0} \sin \theta \cdot x}=2\left[\begin{array}{c}
\cos \left(k_{0} \sin \theta \cdot x\right) \\
\sin \left(k_{0} \sin \theta \cdot x\right)
\end{array}\right],
$$

where $k_{0}$ is the wavenumber and $\theta$ is the incident angle. The resultant electric field has a sinusoidal linearly polarized pattern. Therefore, in the exposure process, azo compounds would rotate to align perpendicular to the electric field, forming a sinusoidal pattern with a phase shift of $\pi$ to the original electric field. It is worth mentioning that Eq. (8) is based on an approximation of small

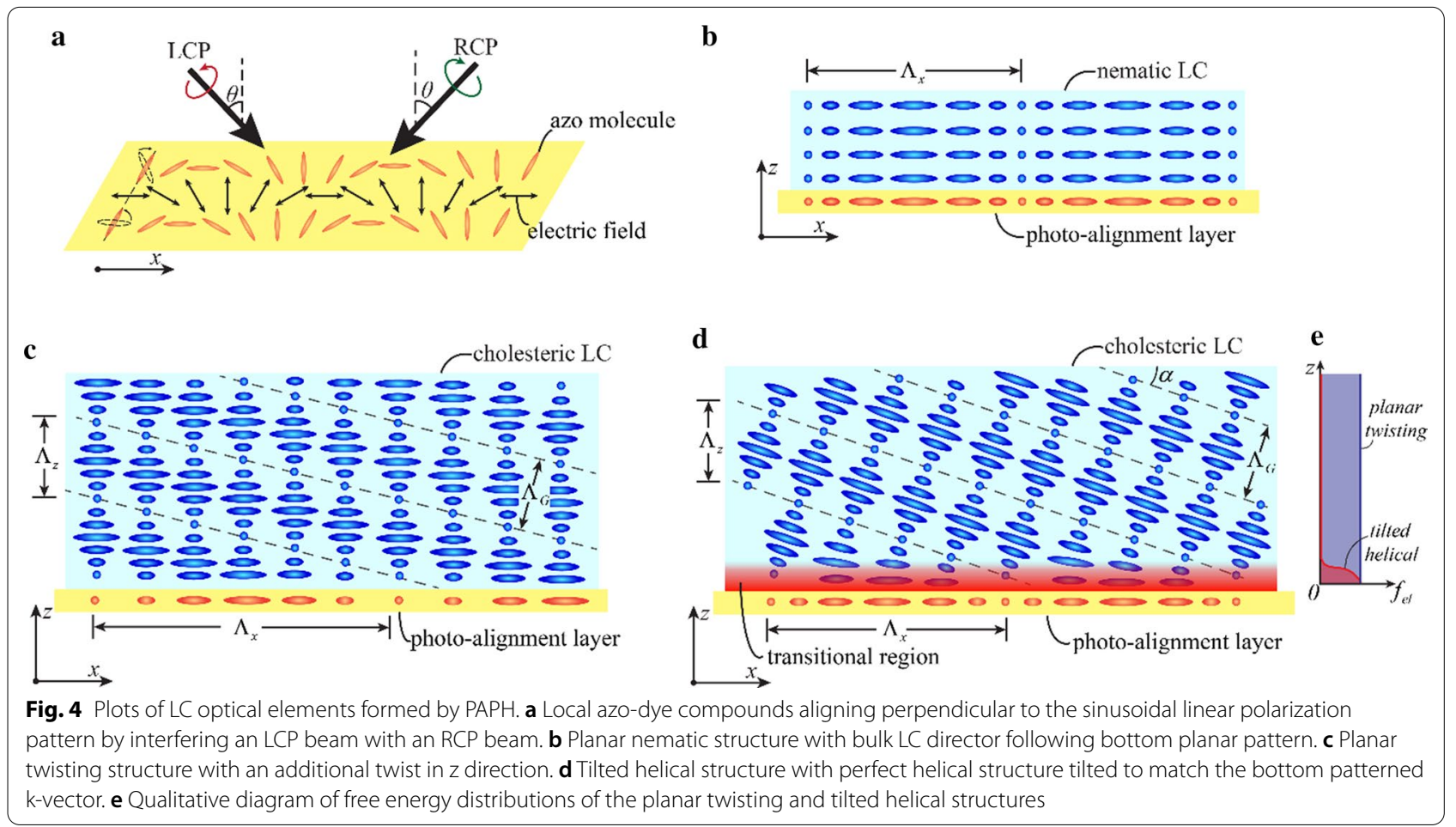


incident angle and ignoring the field component vertical to the substrate. But it has been shown [57] that for large incident angles and even asymmetric incidence, the resultant pattern still has a high degree of linear polarization. Therefore, such a photoalignment pattern recording method can accommodate a reasonably wide range of exposure scenarios.

When a nematic LC layer is deposited on top of the patterned photoalignment layer, the bottom LC directors would follow the sinusoidal pattern, as Fig. 4b depicts. We note this structure as planar-nematic structure. The director $\widehat{\boldsymbol{n}}$ has following form:

$$
\widehat{\boldsymbol{n}}=\left(\sin \left(k_{0} \sin \theta \cdot x\right), \cos \left(k_{0} \sin \theta \cdot x\right), 0\right) .
$$

Plugging Eq. (9) into Eq. (3), we can calculate the elastic free energy density as

$$
f_{e l}=\frac{2 \pi^{2}}{\Lambda_{x}^{2}}\left(K_{1} \cos ^{2} \frac{2 \pi}{\Lambda_{x}} x+K_{2} \sin ^{2} \frac{2 \pi}{\Lambda_{x}} x\right) \approx \frac{2 \pi^{2}}{\Lambda_{x}^{2}} K,
$$

where $\Lambda_{x}=2 \pi /\left(k_{0} \sin \theta\right)$ is the pattern pitch in $x$ direction, and the sign $\approx$ represents the single elastic constant approximation $K_{1}=K_{2}=K_{3}=K$. Although the single elastic constant approximation does not hold true for most LC materials $[58,59]$, it provides us a simple physical insight for an intuitive understanding. The elastic energy is non-zero, indicating such LC director configuration is in a deformed high-energy state. As mentioned earlier, the most relaxed state for nematic LC is a constant director $\widehat{\boldsymbol{n}}$. Therefore, as the thickness of such LC configuration increases, or $\Lambda_{x}$ decreases, the above LC intends to align vertically to form the constant director distribution $[60,61]$. The critical thickness for deformation depends on the elastic constants of the employed LC material [61]. But it is generally in the same order as $\Lambda_{x}$. The LC thickness is usually fixed due to the half-wave retardation condition to be discussed later. Thus, $\Lambda_{x}$ cannot be smaller than a certain value. As a result, such an LC optical element usually exhibits a relatively small diffraction angle $\left(\sim 10^{\circ}\right)$.

Lately, it is found that CLC combined with patterned alignment layer can also form high-quality optical components. If we place a CLC on top of a patterned photoalignment layer, it is natural to assume that the director would follow the bottom pattern, but with a helical twist in $\mathrm{z}$ direction, as shown in Fig. 4c. We call this structure as planar-twisting structure, whose pitch in $\mathrm{z}$ direction $\left(\Lambda_{z}\right)$ is equal to the natural helical pitch of CLC $\left(\Lambda_{C L C}\right)$. Under such condition, the director $\widehat{\boldsymbol{n}}$ has following form:

$$
\widehat{\boldsymbol{n}}=\left(\sin \left(\frac{2 \pi}{\Lambda_{x}} x+\frac{2 \pi}{\Lambda_{z}} z\right), \cos \left(\frac{2 \pi}{\Lambda_{x}} x+\frac{2 \pi}{\Lambda_{z}} z\right), 0\right) .
$$

From Eq. (3), we find the elastic free energy density as:

$$
\begin{aligned}
f_{e l}= & \frac{2 \pi^{2}}{\Lambda_{x}^{2}}\left(K_{1} \cos ^{2}\left(\frac{2 \pi}{\Lambda_{x}} x+\frac{2 \pi}{\Lambda_{z}} z\right)\right. \\
& \left.+K_{2} \sin ^{2}\left(\frac{2 \pi}{\Lambda_{x}} x+\frac{2 \pi}{\Lambda_{z}} z\right)\right) \approx \frac{2 \pi^{2}}{\Lambda_{x}^{2}} K,
\end{aligned}
$$

which has a similar form to Eq. (10).

Recall that the most relaxed CLC state is a helical structure. To deform from planar structure in Fig. 4c to helical structure is indeed very easy, which is different from the case of planar nematic structure. As depicted in Fig. 4d, if the bulk LC directors no longer maintain the planar form and are tilted with an angle $\alpha=\arcsin \left(\Lambda_{G} / \Lambda_{x}\right)$, then a helical structure is obtained, with the LC directors parallel to Bragg surface. The $\mathrm{k}$-vector of bottom pattern is also matched. The Bragg pitch $\Lambda_{G}$ equals to the CLC pitch $\Lambda_{C L C}$ in this case. We note this structure as tilted-helical structure. Both simulations [62] and experiments [62,63] indicate there exists a thin layer of transitional region where LC director evolves from bottom planar pattern to bulk tiltedhelical structure. Remember the helical structure has zero distortion free energy. The free energy density in the transitional layer has a gradient distribution from bottom [whose value is described by Eq. (12)] to top (whose value is zero). Its thickness is dependent on the elastic constants of the employed LC material and anchoring strength of the alignment layer but is typically in the order of $10 \mathrm{~nm}$ according to the simulation results. Therefore, the total free energy of tilted-helical structure is significantly lower than that of planar structure, as shown in Fig. 4e. In this sense, the tilted-helical structure is more stable than the planar-nematic structure in Fig. $4 \mathrm{~b}$. Such a grating can accommodate a very small $\Lambda_{x}$ and therefore a very large diffraction angle.

Although the planar-twisting structure is difficult to exist in a single-layer bulk LC, it can be fabricated with multiple spin-coating procedures. The thickness of each spin-coated layer should be small enough so that the planar form is maintained. Another limiting case is when the chiral concentration is low and therefore $\Lambda_{C L C}$ is very large. This case is similar to the planar-nematic structure, at a semi-stable state with a high distorted free energy.

\subsection{Device fabrication}

From above discussions, PAPH basically includes two parts: (1) patterning photoalignment layer and (2) deposition of LC material. For the patterning part, our previous discussion focuses on the interference of LCP and RCP beams, which can be achieved using several types of interferometers $[51,55,64,65]$. Figure 5 a shows a typical interferometer 
for recording an off-axis lens pattern. To record an arbitrary pattern, we need to firstly fabricate such a template using methods like diamond turning. Then we can relay the wavefront of that template onto the sample [51].

In addition to interferometers, several other methods can also be employed to produce linearly polarized pattern, such as spatial light modulator (SLM) [66-68], direct laser scanning technique [69-71], and replication mask [72-75]. The SLM approach relies on local modulation of phase retardation to control the final linear polarization direction of each pixel, as depicted in Fig. 5b. The device resolution is limited by the SLM and therefore it can only produce pattern with a relatively large period. The direct laser scanning technique uses a laser whose linear polarization direction is controlled by a polarization rotator. Such a polarization rotator is synchronized with the translation stage to write a spatial pattern in a point-to-point manner, as shown in Fig. 5c. Aside from relatively slow writing speed, it may also face the resolution issue because the smallest feature size is limited by the focused laser spot size, which generally has the same order as the laser wavelength. However, to achieve a large diffraction angle, the corresponding pattern feature size should be much smaller than the wavelength. Finally, the patterned polarizer method directly converts the input circularly polarized or unpolarized light to patterned linear polarization with a mask, as sketched in Fig. 5d. The replication mask can be a polarizer mask fabricated by photolithography $[72,73]$ or interferometer based PAPH
[75]. Photolithography can achieve a sub-wavelength feature size but to write a large-scale $(\sim \mathrm{cm})$ sample is time consuming. Alternatively, the mask can also be a waveplate mask [74] fabricated through all other methods. It is worth mentioning that the replication mask method has the highest potential for mass production, which is critical for applications like VR and AR.

The LC material deposition can be categorized into spin-coating and cell formation. In spin-coating, a reactive mesogen solution is overcoated on top of the bottom photoalignment layer. After solvent evaporation, a UV light is used to polymerize the reactive mesogen. This process can be repeated several times. Each time, the top surface of previous layer serves to align the subsequent layer. Such a multiple spin-coating routine can be used to form some specific structures that would otherwise be distorted in a single layer approach [76, 77], as discussed above. On the other hand, the cell formation method uses two substrates to form a cell. The photoalignment layer is placed on one or both inner surfaces of the substrates. A fluidic LC is then infiltrated into the cell and form the optical element. If the substrates are coated with indium tin oxide electrodes, the LC directors can be switched by an external voltage. Such an LC optical element is called active device, while the LC polymer based optical element is called passive device. The cured LC polymer is not switchable. To utilize its polarization selectivity, we need to add an active polarization rotator to control the input polarization.

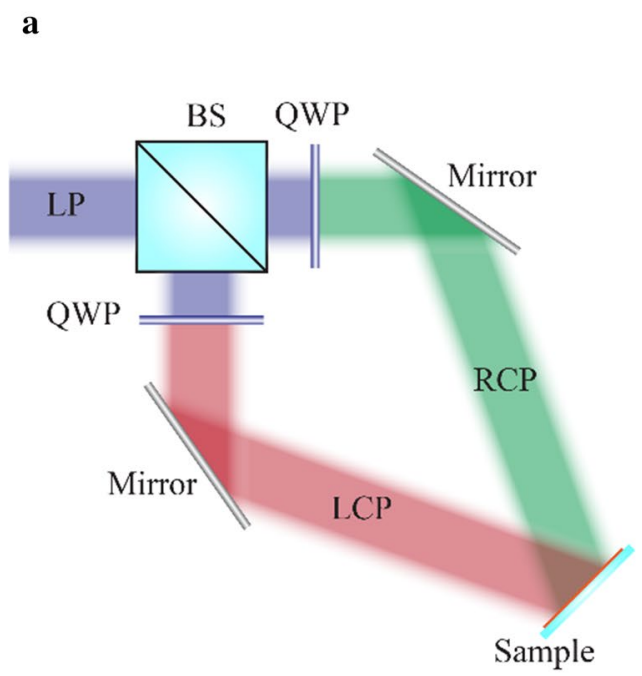

b

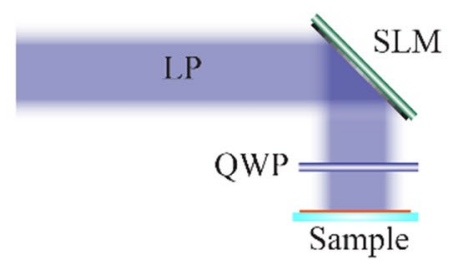

c

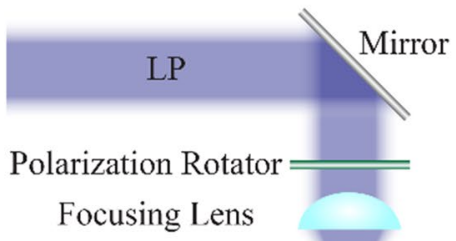

d
Sample
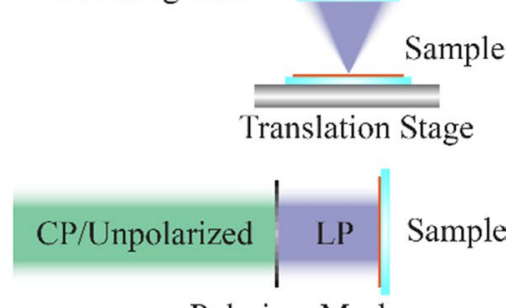

Polarizer Mask

Fig. 5 Methods to produce patterned linear polarization. a An interferometer to record an off-axis lens pattern. b SLM-based method. c Laser scanning method. d Patterned polarizer mask method. BS: beam splitter. LP: linear polarization. QWP: quarter-wave plate. CP: circular polarization 


\subsection{Response under applied voltage}

In the planar-nematic structure, if the employed LC material has a positive dielectric anisotropy $(\Delta \varepsilon>0)$, then we can dynamically switch the cell with an electric field along $\mathrm{z}$ direction. From Eq. (10), the LC director has the tendency to reorient perpendicularly to lower the free energy. Similar to the Fréedericksz transition in a homogeneous cell, there is a threshold voltage $\left(V_{t h}{ }^{\prime}\right)$ over which the planar director starts to deform. With some approximations [61], $V_{t h}{ }^{\prime}$ can be expressed as

$$
V_{t h}^{\prime}=\pi \sqrt{\frac{K_{1}}{\varepsilon_{0} \Delta \varepsilon}} \sqrt{1-\left(\frac{d}{d_{c}}\right)^{2}}=V_{t h} \sqrt{1-\left(\frac{d}{d_{c}}\right)^{2}},
$$

where $V_{t h}$ is the threshold voltage of a homogeneous cell and $d_{c}$ is the critical thickness. From Eq. (13), $V_{t h}{ }^{\prime}$ is lower than $V_{t h}$. This can be explained by the high-energy state of the planar nematic structure. As discussed above, the LC directors have non-zero free energy and tend to align vertically, which is the same as electric field direction. Therefore, the LC directors in a planar twisting structure are easier to deform than those of a homogeneous cell. The response time has the same expression as a homogeneous cell [78]:

$$
\begin{gathered}
\tau_{\text {rise }}=\frac{\tau_{0}}{\left|1-\left(V / V_{t h}\right)^{2}\right|}, \\
\tau_{\text {decay }}=\tau_{0}=\frac{\gamma_{1} d^{2}}{K_{1} \pi^{2}},
\end{gathered}
$$

where $\tau_{\text {rise }}$ and $\tau_{\text {decay }}$ represents the rise time and free relaxation time, respectively, $\gamma_{1}$ is the rotational viscosity, $d$ is the cell gap, and $V$ is the applied voltage. By choosing a low viscosity $\mathrm{LC}$ material and thin cell gap, the response time can reach $1-2 \mathrm{~ms}$.

In a tilted helical structure, the LC dynamic response is more complicated $[62,79]$. Although the electric field free energy is lower for the LC directors in $\mathrm{z}$ direction, the elastic free energy is high when all the $\mathrm{LC}$ directors are aligned vertically. According to numerical simulation and experiments, there is also a threshold voltage over which the helical structure begins to rotate toward $\mathrm{z}$ direction. But the pattern period in $x$ direction is still fixed and therefore the diffraction angle does not change. At a certain point, the Bragg surface will be aligned vertically, which has the same configuration as a uniform lying helix (ULH) structure. As the voltage further increases, the ULH structure will be deformed and unwinded, until all the LC directors are aligned vertically.

\section{Optical properties}

Although versatile optical elements, such as gratings, on/ off-axis lenses, and freeform structures can be fabricated with above LC structures, the local region of these elements can be treated as a grating. Therefore, we will focus on gratings in this section. Before we dive into the detailed grating structures, it is necessary to introduce the optical simulation methods first.

\subsection{Simulation methods}

To categorize a grating, the so-called $\mathrm{Q}$ factor [80] $Q=2 \pi \lambda d / \bar{n} \Lambda_{G}^{2}$ can be used. It generally describes the degree of modulation of a grating. When $\mathrm{Q}<1$, the modulation is not strong and such a grating is referred as RamanNath grating. When $\mathrm{Q}>1$, the modulation is strong, and we call such a grating as Bragg grating.

For Raman-Nath gratings, in a small local region, the structure can be regarded as uniform in $x$ direction, but can be nonuniform in $z$ direction, as Fig. 6a shows. Therefore, we can use 4-by-4 matrix method [78] to calculate the output electric field for each small local region. The basic idea of 4-by-4 matrix method is to analytically solve Maxwell's equations in the uniform anisotropic region and use the boundary conditions to calculate fields. Between two consecutive interfaces, the Maxwell's equations can be formulated as following form by eliminating the z-direction field components:

$$
\frac{\partial}{\partial z}\left[\begin{array}{c}
E_{x} \\
E_{y} \\
H_{x} \\
H_{y}
\end{array}\right]=i k_{0} \boldsymbol{R}\left[\begin{array}{c}
E_{x} \\
E_{y} \\
H_{x} \\
H_{y}
\end{array}\right]
$$

where $k_{0}$ is the wavenumber, $\boldsymbol{R}$ is a 4 -by- 4 matrix, $E_{x, y}$ and $H_{x, y}$ are the tangential electric and magnetic fields. Equation (16) can be easily solved by diagonalizing matrix $\boldsymbol{R}$. Therefore, we can correlate tangential fields at two consecutive interfaces by

$$
\begin{aligned}
& {\left[\begin{array}{c}
E_{x} \\
E_{x} \\
H_{x} \\
H_{x}
\end{array}\right]_{i+1}=\boldsymbol{T}\left[\begin{array}{llll}
\mathrm{e}^{i k_{0} r_{1} d_{i}} & & & \\
& \mathrm{e}^{i k_{0} r_{2} d_{i}} & & \\
& & \mathrm{e}^{i k_{0} r_{3} d_{i}} & \\
& & & \mathrm{e}^{i k_{0} r_{4} d_{i}}
\end{array}\right]} \\
& \boldsymbol{T}^{-1}\left[\begin{array}{c}
E_{x} \\
E_{x} \\
H_{x} \\
H_{x}
\end{array}\right]_{i}=\boldsymbol{P}_{i}\left[\begin{array}{c}
E_{x} \\
E_{x} \\
H_{x} \\
H_{x}
\end{array}\right]_{i}
\end{aligned}
$$

where $r_{i}$ are the eigenvalues of $\boldsymbol{R}$ and $\boldsymbol{T}$ is the transformation matrix. Thus, we can correlate the tangential fields at input and output interfaces by multiplication of matrices $\boldsymbol{P}_{i+}$ Finally, the reflected $\overrightarrow{\boldsymbol{E}}_{0}$ and transmitted electric field $\overrightarrow{\boldsymbol{E}}_{N}$ components can be calculated by a conversion matrix between $\left[\overrightarrow{\boldsymbol{E}}^{+}, \overrightarrow{\boldsymbol{E}}^{-}\right]$and $[\overrightarrow{\boldsymbol{E}}, \overrightarrow{\boldsymbol{H}}]$. 


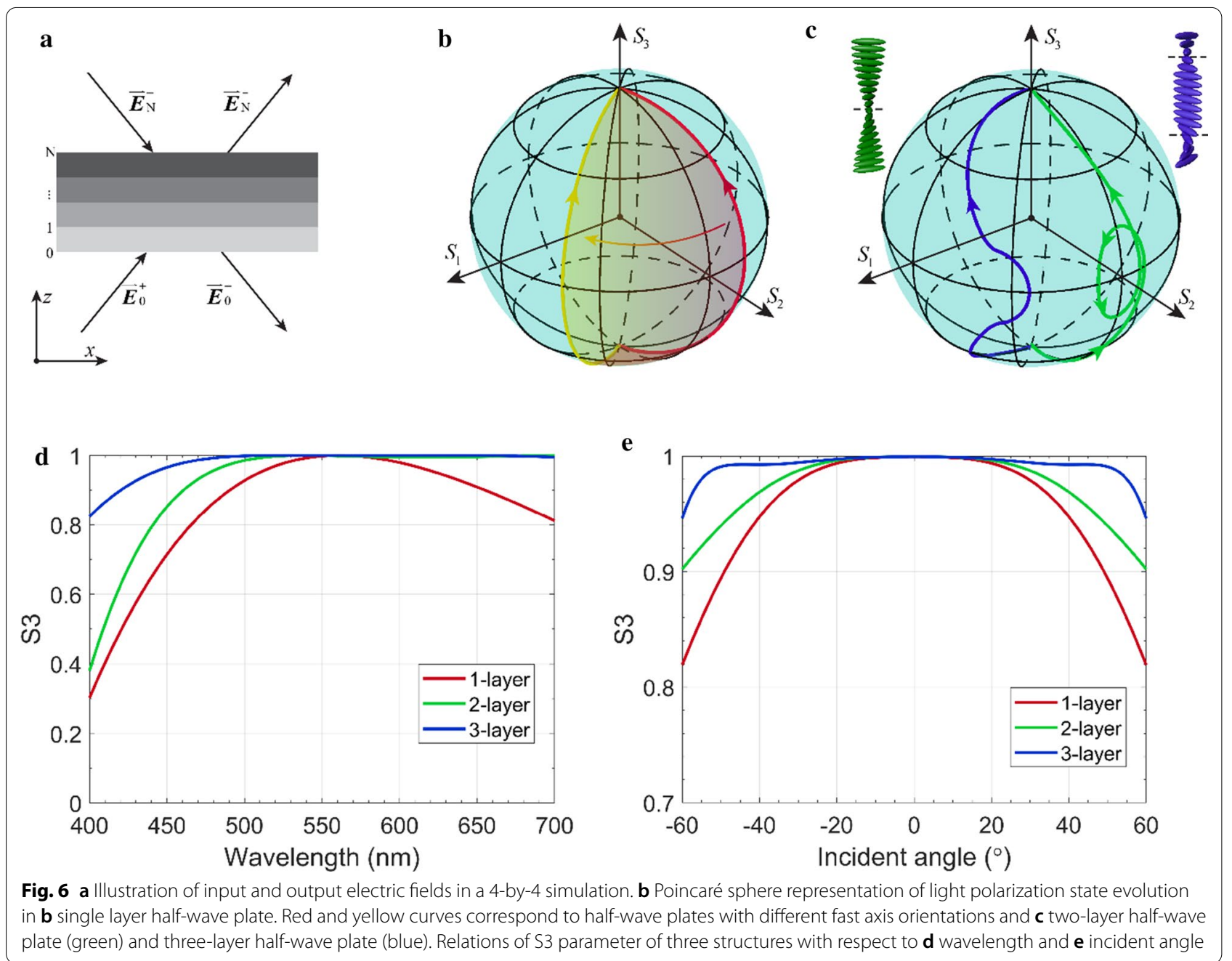

For Bragg gratings with a small pitch in $\mathrm{x}$-direction, the above approximation on the $\mathrm{x}$-direction uniformity is no longer valid. Because the grating pitch is small, the propagating light already experiences non-uniformity in $\mathrm{x}$-direction when passing the medium. A more precise simulation method called rigorous coupled-wave analysis (RCWA) [81-83] should be adopted. In RCWA, the electric and magnetic fields are expressed in Fourier expansions, according to the grating period in $\mathrm{x}$ directions. The formulation of RCWA, interestingly, almost has the same procedure as the 4-by-4 method mentioned above, by replacing scalar values of $E(H)_{x, y}$ in Eq. (16) with vectors $\overrightarrow{\boldsymbol{E}}(\overrightarrow{\boldsymbol{H}})_{x, y}$ corresponding to Fourier coefficients. Details can be found in [81]. RCWA can therefore be regarded as a "4M-by-4M" method with $\mathrm{M}$ being the order of Fourier expansion. Of course, there are other simulation methods like finite element method (FEM) and finite-difference time-domain (FDTD). But for simulation of LC gratings, RCWA has a significantly faster speed and higher precision [81].

\subsection{Transmissive LC grating}

Let us first look at transmissive LC gratings. The simplest case is the planar-nematic structure with grating thickness satisfying half-wave retardation. The grating can therefore be regarded as a patterned half-wave plate with spatially rotating optical axis. With an incident circularly polarized light, Jones matrix can be expressed as

$$
\begin{aligned}
J_{H W P}\left[\begin{array}{c}
1 \\
\pm i
\end{array}\right] & =\left[\begin{array}{cc}
\cos \left(\frac{2 \pi}{\Lambda_{x}} \cdot x\right) & \sin \left(\frac{2 \pi}{\Lambda_{x}} \cdot x\right) \\
\sin \left(\frac{2 \pi}{\Lambda_{x}} \cdot x\right) & -\cos \left(\frac{2 \pi}{\Lambda_{x}} \cdot x\right)
\end{array}\right] \\
{\left[\begin{array}{c}
1 \\
\pm i
\end{array}\right] } & =\left[\begin{array}{c}
1 \\
\mp i
\end{array}\right] e^{ \pm i \frac{2 \pi}{\Lambda_{x}} \cdot x}
\end{aligned}
$$

where $\Lambda_{x}$ is the grating pitch. From Eq. (18), we can see the handedness of the circularly polarized light is flipped, while adding a linear phase term, corresponding to the diffraction of output light. This type of grating is usually called geometric phase grating or Pancharatnam-Berry (PB) grating. The term is related to the cyclic evolution 
of light polarization state in two different trajectories with same starting and ending states. Although the ending polarization state is same for two trajectories, there is an additional phase difference due to the evolving path difference. The concept can be visualized with Poincaré representation [78, 84]. Using 4-by-4 method, we can calculate the light polarization state at a specific thickness and plot it in the Poincaré sphere.

As shown in Fig. $6 b$, when starting from LCP $(S 3=-1)$ state, the light goes through a big arc and reaches RCP state $(\mathrm{S} 3=+1)$. The two different paths (red and yellow) correspond to two half-wave plates with different azimuthal orientations. The phase difference between two ending RCP states is proportional to the area between the two paths.

The planar-nematic state has the simplest configuration as a PB grating. However, its efficiency is also vulnerable to the change in wavelength or incident angle. This can be explained by a deep observation of the path on Poincaré sphere. Because the path is the shortest one from south to north pole, a deviation in path length from the change of wavelength or incident angle would directly result in a departure of ending point from north pole. This is because the "shortest" path has little tolerance over the path length change. For practical applications, however, a large tolerance on wavelength and incident angle is generally desired. To improve the angular and spectral performances, we can design a multi-layer structure $[52,53,85,86]$. In each layer, the LC director can have a twist along surface normal, which has the same structure as the planar-twisting one, plotted in Fig. 4c. It should be clarified that the pitch in $\mathrm{z}$ direction in this case is usually large, so the associated distortion free energy is small. The planar-twisting structure can be maintained. As an example, let us examine a 2-layer structure and a 3-layer structure, as shown in Fig. 6c. Both structures have symmetric configurations. The 2-layer structure has a twisting angle of $70^{\circ}$ for the first layer and $-70^{\circ}$ for the second layers, with a total thickness of $3.4 \mu \mathrm{m}$. The 3-layer structure has a twisting angle of $70^{\circ}, 0^{\circ},-70^{\circ}$ and thickness of $1.0 \mu \mathrm{m}, 1.4 \mu \mathrm{m}, 1.0 \mu \mathrm{m}$ for each layer. The LC birefringence is 0.17 . The paths on Poincare sphere are plotted in Fig. 6c. Both paths are deformed and prolonged. The angular and spectral responses are plotted in Fig. 6d, e, respectively. For a single layer, S3 drops quickly as the wavelength deviates from the central wavelength of $550 \mathrm{~nm}$. But the 2-layer structure can maintain a S3>0.95 in the $500 \mathrm{~nm}$ to $700 \mathrm{~nm}$ spectral range. The 3-layer structure further extends the spectral range from 450 to $700 \mathrm{~nm}$, which covers a large portion of the visible spectrum. Regarding angular response, the 2-layer structure can maintain $\mathrm{S} 3>0.9$, while the 3-layer structure can maintain S3 $>0.95$ for the incident angle within $\pm 60^{\circ}$.
Although the 4-by-4 matrix method provides a clear understanding of PB gratings, it is based on an assumption of large $\Lambda_{x}$. For precise calculation and optimization, RCWA is still required. The optimized structure by RCWA may deviate from that from 4-by-4 method. Still, 4-by-4 method can serve as an analytical tool and set starting values for RCWA optimization. As $\Lambda_{x}$ gets smaller, the PB grating gradually falls into Bragg regime. At this point, the planar-nematic structure may face the alignment issue as previously discussed. To overcome this, we can use multiple spin-coatings, with thickness of each layer below the critical thickness of deformation. However, this approach also raises another issue. When $\Lambda_{x}$ becomes small enough, the normally incident light is already deflected in the grating region, so the light sees change of LC orientation as it propagates in $\mathrm{z}$ direction. This leads to a relatively low efficiency at normal incidence. To improve normal incidence efficiency, we have to adopt the planar-twisting structure to manually rotate Bragg surface so that the deflected light is propagating along Bragg surface and therefore experiencing no change in LC directors. But this also means we have to adopt multiple spin-coatings to suppress the tiltedhelical structure. The required thickness for each layer should be thinner than the transitional layer in tiltedhelical structure, which makes the practical fabrication remarkably difficult.

To elucidate the design procedures using RCWA, we simulate two transmissive gratings: one Raman-Nath grating with $\Lambda_{x}=4 \mu \mathrm{m}$ and one Bragg grating with $\Lambda_{x}=500 \mathrm{~nm}$. The angle between Bragg surface and surface normal is $20^{\circ}$. Both gratings have a thickness of $1.7 \mu \mathrm{m}$. The fast computation of RCWA enables us to vary both incident angle and wavelength to observe the change in diffraction efficiency, which normally takes weeks for FEM or FDTD methods. The results are shown in Fig. 7a, b. We can see due to the tilted Bragg surface, the normal incidence in Bragg grating has a high efficiency. Without the tilting, the high efficiency peak would shift to $-20^{\circ}$ incidence. The high-efficiency band in angular dimension is much narrower for Bragg grating than Raman-Nath grating. To enlarge spectral bandwidth, theoretically we can stack different layers of planar-twisting structure together, but that further increases the fabrication difficulty.

\subsection{Reflective LC grating}

Reflective LC gratings have very different optical properties and fabrication requirements from their transmissive counterparts. In order to diffract light to reflective orders, the grating pitch is usually small. Therefore, reflective LC gratings are generally Bragg gratings. We will focus on the tilted-helical structure due to its easier 

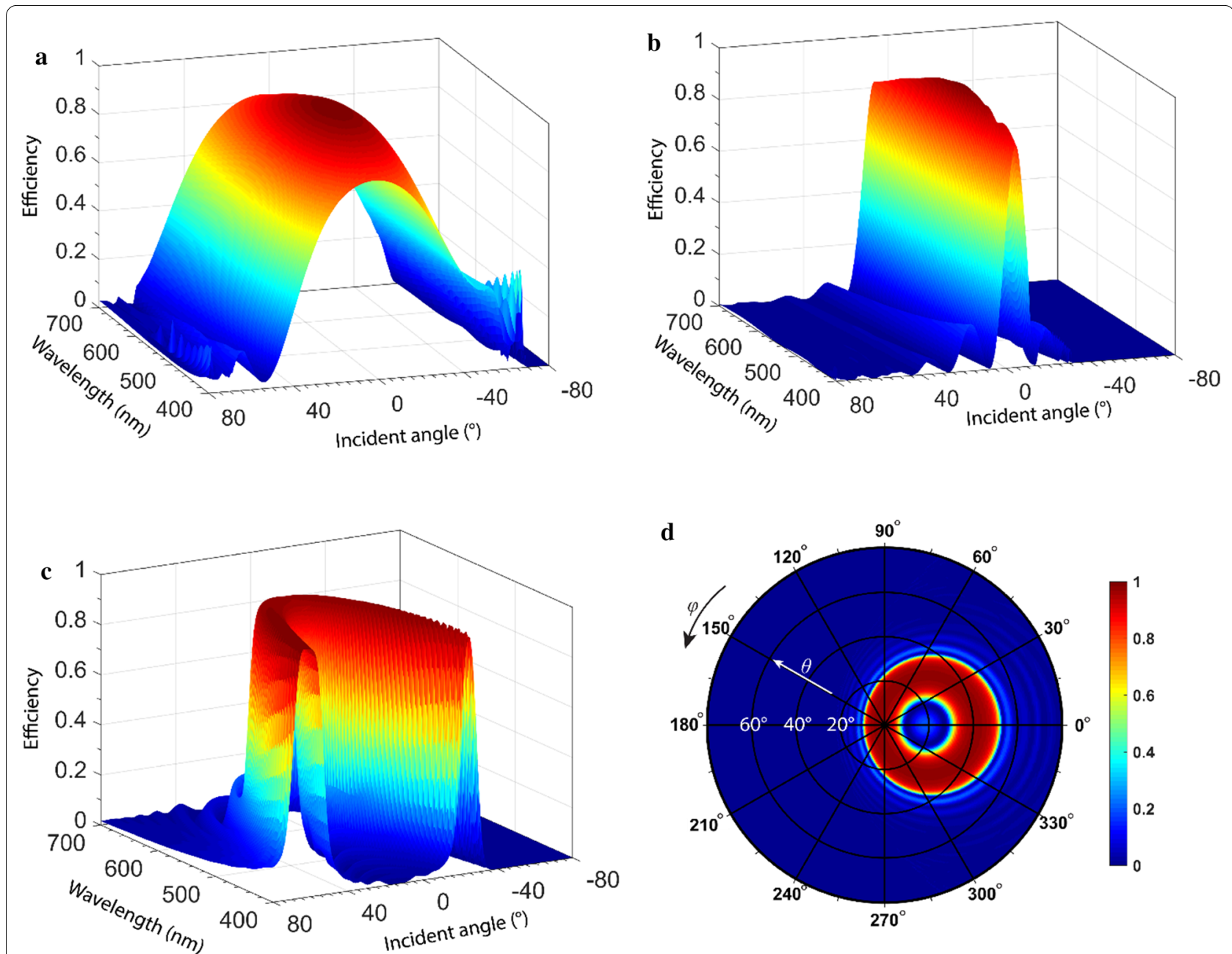

Fig. 7 Simulated results with RCWA. Plot of efficiency with relations to wavelength and incident angle for a Raman-Nath transmissive grating, $\mathbf{b}$ Bragg transmissive grating, and $\mathbf{c}$ Bragg reflective grating. $\mathbf{d}$ Plot of efficiency with relations to incident polar and azimuthal angles of the Bragg reflective grating

fabrication than the planar-twisting structure. As an example, we chose a grating with $\Lambda_{G}=190 \mathrm{~nm}$ and tilt angle between Bragg surface and substrate is $\alpha=20^{\circ}$. The efficiency in relation with incident angle and wavelength is plotted in Fig. 7c. Unlike Bragg transmissive grating with a straight high-efficiency band, the reflective grating has a "U-shape" band with a cut-off wavelength. In our case, the cut-off wavelength is around $620 \mathrm{~nm}$. Over the cut-off wavelength the efficiency quickly drops to zero regardless of the incident angle. We can obtain the spectral plot by sectioning the U-shape band in Fig. 7c along the incident angle. For a specific incident angle, there is always only one peak in the spectral plot. The angular plot can be acquired similarly by sectioning the U-shape band along a specific wavelength. The angular plot, however, could have one or two peaks, depending on the wavelength. For example, for the wavelength of $400 \mathrm{~nm}$, the angular plot has two peaks. But when the wavelength is close to the cut-off wavelength, for example, at $600 \mathrm{~nm}$, there is only one peak in the angular plot. To understand the angular response more clearly, we will consider the general conical diffraction where both incident polar angle $\theta$ and azimuthal angle $\phi$ change. The result for $\lambda=550 \mathrm{~nm}$ is plotted in Fig. $7 \mathrm{~d}$. The high-efficiency band has a donut shape. With a certain range of incident angles in center exhibit low efficiency. When $\phi=0^{\circ}$, that is, when we draw a horizontal line and see the intersection, the efficiency- $\theta$ plot corresponds to the two-peak case of Fig. 7c. As the wavelength increases to a certain range, the hole in "donut" disappears and the whole band is connected, with the shape of a disk. Further details of optical properties of various types of LC optical elements can be found in [81]. 
Finally, it is necessary to discuss the polarization dependency of above LC gratings. For a transmissive PB grating, as seen from Eq. (18), the output phase is opposite for the incident LCP and RCP lights. As a result, the diffraction angle is opposite, as Fig. 8a depicts. The handedness is also flipped after the PB grating. Although the $\mathrm{PB}$ grating is passive, we can add an active half-wave plate to control the incident polarization (LCP or RCP) so that we can switch the diffraction order. A twisted-nematic LC cell with a quarter-wave plate is a commonly used broadband polarization rotator [87]. For binary switching, its response time can be as fast as $1-2 \mathrm{~ms}$, depending on the LC material and cell gap employed. If we stack multiple of these devices together, we can achieve a beam steerer with multiple but discrete steering angles [88]. Transmissive PB lenses can also be fabricated, by replacing one collimated beam with a divergent beam in the interferometer. Opposite phase profiles for LCP and RCP in this case means opposite optical powers, one corresponding to converging lens and the other diverging, as shown in Fig. 8b. The combination of a PB lens and a polarization converter results in a bifocal lens. If we stack multiple bifocal lenses together and carefully choose the lens' optical powers, we can obtain a quasi-continuous tunable lens. For reflective LC optical elements, the polarization dependency is different. If the incident circularly polarized light, say LCP, has the same handedness as the CLC, then diffraction occurs. While for RCP, there is no diffraction. Various types of reflective optical elements can be fabricated, such as grating, reflective onaxis lens or off-axis lens, as illustrated in Fig. 8c-e. When combined with a polarization-modulating device, reflective gratings or lenses can also achieve different types of functionalities.

\section{Applications}

\subsection{Virtual reality displays}

Most of current VR displays adopt a simple optical layout with a display panel and a collimation lens, as shown in Fig. 9. The image from a high-resolution display panel (LCD, OLED, or micro-LED) is relayed to a far distance. Two different images are delivered to the viewer's left and right eyes. The spatial parallax of images induces 3D perception in the viewer's brain. Three factors in a VR display play important roles: resolution density, form factor, and vergence accommodation conflict (VAC)

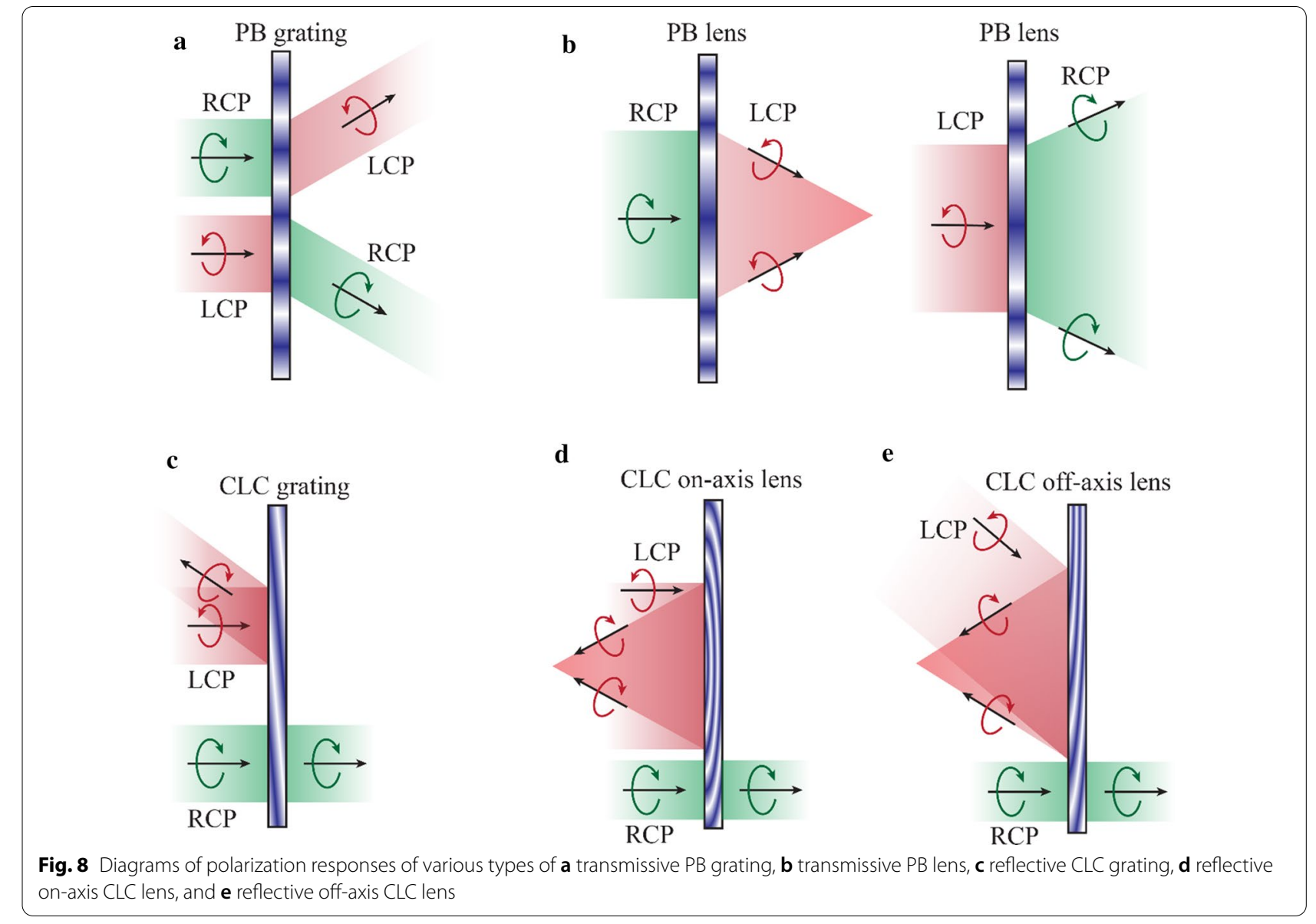




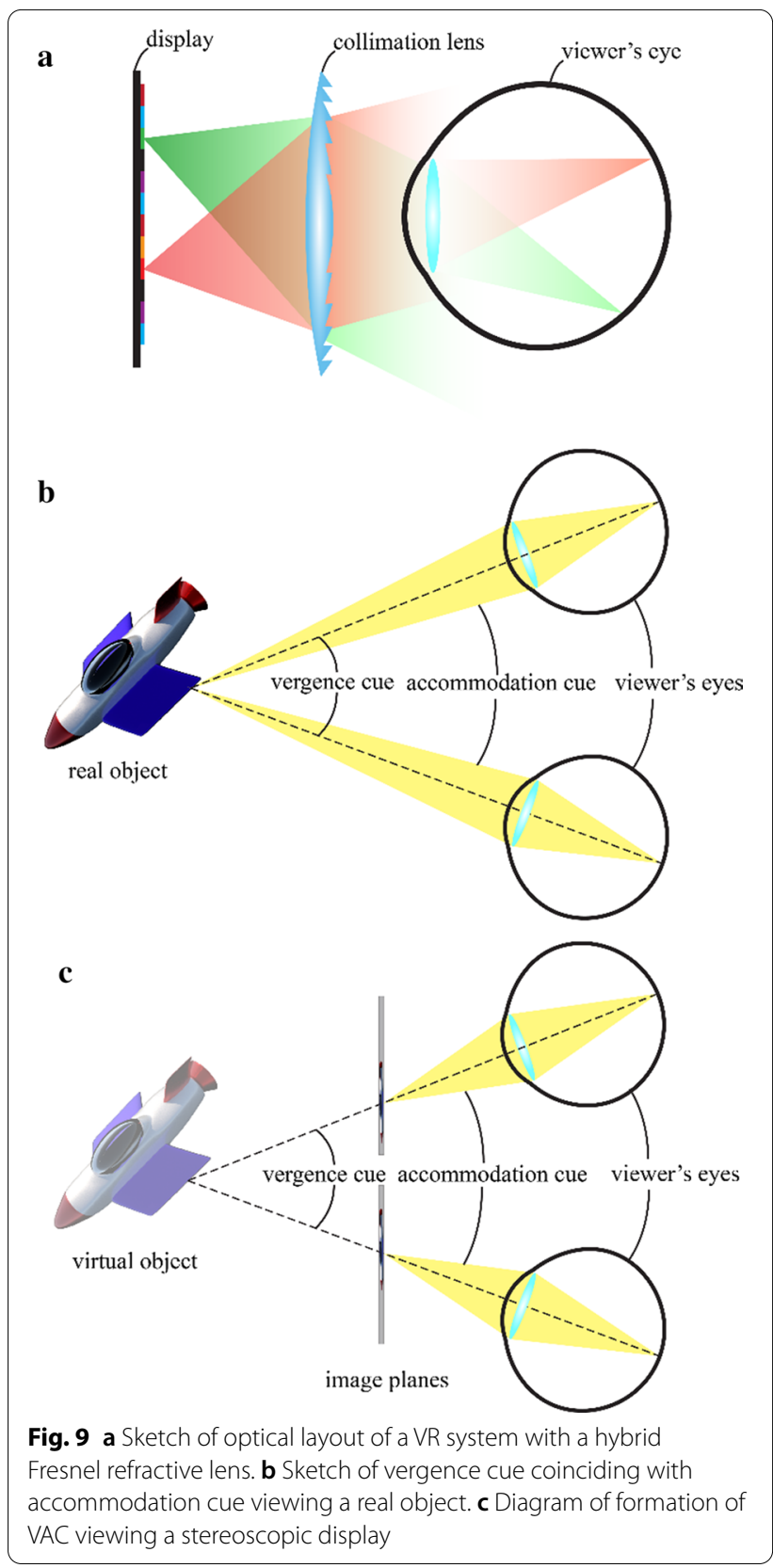

[9]. Resolution density determines the pixels per degree (PPD) perceived by the viewer. For a normal eye, the visual acuity is 1 arcminute, which translates to PPD $=60$. To achieve $100^{\circ} \mathrm{FoV}$, the display resolution should be $6 \mathrm{~K}$ [89]. An inadequate resolution density would cause the annoying screen-door effect. At present stage, most of the VR displays have not reached this target yet, but the resolution density is steadily increasing. Therefore, here we will mainly focus on form factor and VAC. Form factor is mainly determined by the total track from display panel to eyebox. The distance from collimation lens to display panel is usually equal to the lens focal length. Further shrinking the focal length brings issues of aberrations. VAC results from the stereoscopic nature of display. It causes nausea and visual discomfort to users and is considered a major obstacle for VR.

The formation of VAC is plotted in Fig. 9b, c. There are two main cues in human's 3D perceptions: vergence cue and accommodation cue. Vergence cue comes from the relative rotation of two eyes to overlap left and right images in viewer's perception. Accommodation cue comes from focusing of eye lens to get a sharp image of object. When viewing a real $3 \mathrm{D}$ object, as depicted in Fig. 9b, both cues fall on the object and there is no conflict. But in an optical system plotted in Fig. 9a, the distance of image plane is fixed and so is the accommodation cue. When the intended distance of virtual 3D object is different from image plane, the induced vergence cue is different from accommodation cue. Such a mismatch is referred as VAC.

To reduce form factor, polarization-based folded optics, also known as "pancake" optics can be used [90, 91]. A typical layout of pancake optics is plotted in Fig. 10a. Let us assume the display panel emits a linearly polarized light (s-wave). First, the s-wave passes through the half-mirror and is reflected by the reflective polarizer. Then it is reflected again by the half-mirror, which is integrated with a quarter-wave plate (not shown in the drawing). The light is converted to $\mathrm{p}$-wave and passes through the reflective polarizer. It should be noted the quarterwave plate can also be integrated onto the reflective polarizer. The optical path in between the half-mirror and the reflective polarizer is three-folded. However, the conventional pancake optics still requires some refractive elements, which adds to system weight and form factor. The total system efficiency is relatively low (25\%) mainly because of the transmission and reflection loss from the half-mirror.

If we replace the reflective polarizer with a flat CLC lens as plotted in Fig. 10b, then the form factor can be dramatically improved. In this case, all the optical power is provided by the CLC lens. Alternatively, we may replace the half-mirror with a holographic lens fabricated by, for example, holographic exposure of photopolymer. Then the holographic lens can provide an extra optical power to compensate for aberrations. The different behaviors of light on holographic lens, as indicated by A and $\mathrm{B}$, are due to the strong angular selectivity of hologram. The replacement of half-mirror also improves the system efficiency to nearly $100 \%$. But the strong spectral angular selectivity of holographic lens necessitates a monochromatic laser light source.

Another important issue, VAC, can also be well addressed with LC flat optics [92-95]. As previously 
a

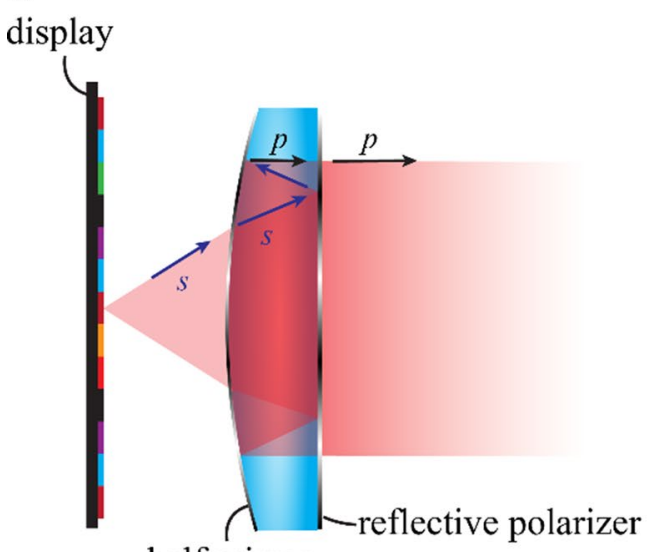

b

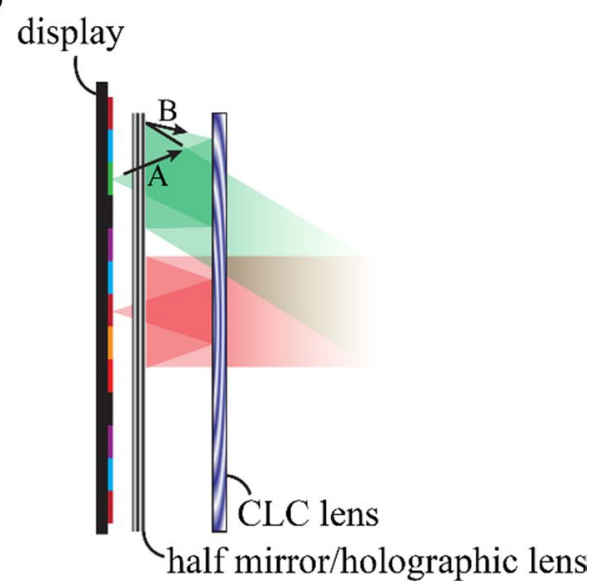

Fig. 10 a Schematics of a pancake optical system for VR using a refractive lens, reflective polarizer and half mirror. b Sketch of a thin pancake optical VR display with a flat CLC lens and a half mirror/holographic lens

discussed, the polarization dependent focusing power and electrically switchable property of PB lens enable us to fabricate a quasi-continuous tunable LC lens. As sketched in Fig. 11a, the quasi-continuous tunable LC lens module consists of $\mathrm{N}$ sets of $\mathrm{PB}$ lens and polarization converter modules. If the $\mathrm{PB}$ lens is a passive device (made of LC polymer), then each lens set can produce 2 optical powers, $\pm \mathrm{D}$. The quasi-continuous tunable LC lens module should have a total of $2^{\mathrm{N}}$ focal powers. If the PB lens itself is active (made of LC cell), then the lens set can produce 3 optical powers, $0, \pm \mathrm{D}$. The lens module should have $3^{\mathrm{N}}$ focal powers. For estimate, a stack of $6 \mathrm{bi}$ focal lenses will produce 64 focal powers, which should be sufficient for a smooth transition. Compared to other tunable lenses, such as liquid lens or deformable lens, the quasi-continuous tunable LC lens has advantages in fast response time $(\sim \mathrm{ms})$, large aperture, and small optical aberrations.

As described earlier, the issue of VAC mainly originates from the fixed image plane and therefore accommodation cue. With quasi-continuous tunable LC lens, the image plane is no longer fixed, driving accommodation cue to coincide with vergence cue. Regarding how to drive the focal plane, generally two approaches can be adopted. One is vari-focal and the other is multi-focal, as depicted in Fig. 11b, c. In a vari-focal approach, the tunable lens only has one focal plane in each time frame, which has the same depth as the viewer's vergence cue (e.g., object 1 ). This implies the system needs to know the viewer's vergence in advance and therefore an eye tracker system is required. The eye tracker recognizes the viewer's pupil locations of both eyes and calculate the vergence cue. Then the tunable lens chooses the closest depth to display a 2D image. However, in our natural viewing experience, any object (e.g., object 2) not at our gazed depth should be blurred. Therefore, we have to digitally blur the corresponding contents in the 2D image to make it look natural.

The multi-focal approach produces multiple depths at each time frame. The purpose is to restore the 3D light field by approximating it with multiple planes. For human vision, it is analyzed that around 6 planes should be enough to construct a high-quality light field [96]. The main question to be answered is how to determine the position and content of each plane. This involves the optimization of light field $[97,98]$, which often requires a heavier computation for a better image quality. In principle, with an adequate number of planes, eye tracking is no longer required. But in the case of inadequate number of planes, eye tracking helps improve the image quality [99]. As for how to produce multiple depths with the quasi-continuous tunable lens, time-multiplexing [93] or polarization-multiplexing [94] can be used. In timemultiplexing, the quasi-continuous tunable lens simply switches among intended multiple depths in a single time frame. But the display should also update its content in synchronization, which lowers the final frame rate of system by the number of depths. Polarization-multiplexing requires a pixel-level polarization management and does not require synchronization of display. It therefore does not sacrifice frame rate. However, it only supports two depths. But when combined with time-multiplexing, it can mitigate the frame rate sacrifice by half.

Finally, it is worth mentioning although the PB lens can well address VAC, it also brings potential issue of chromatic aberration due to its intrinsic diffractive property. 


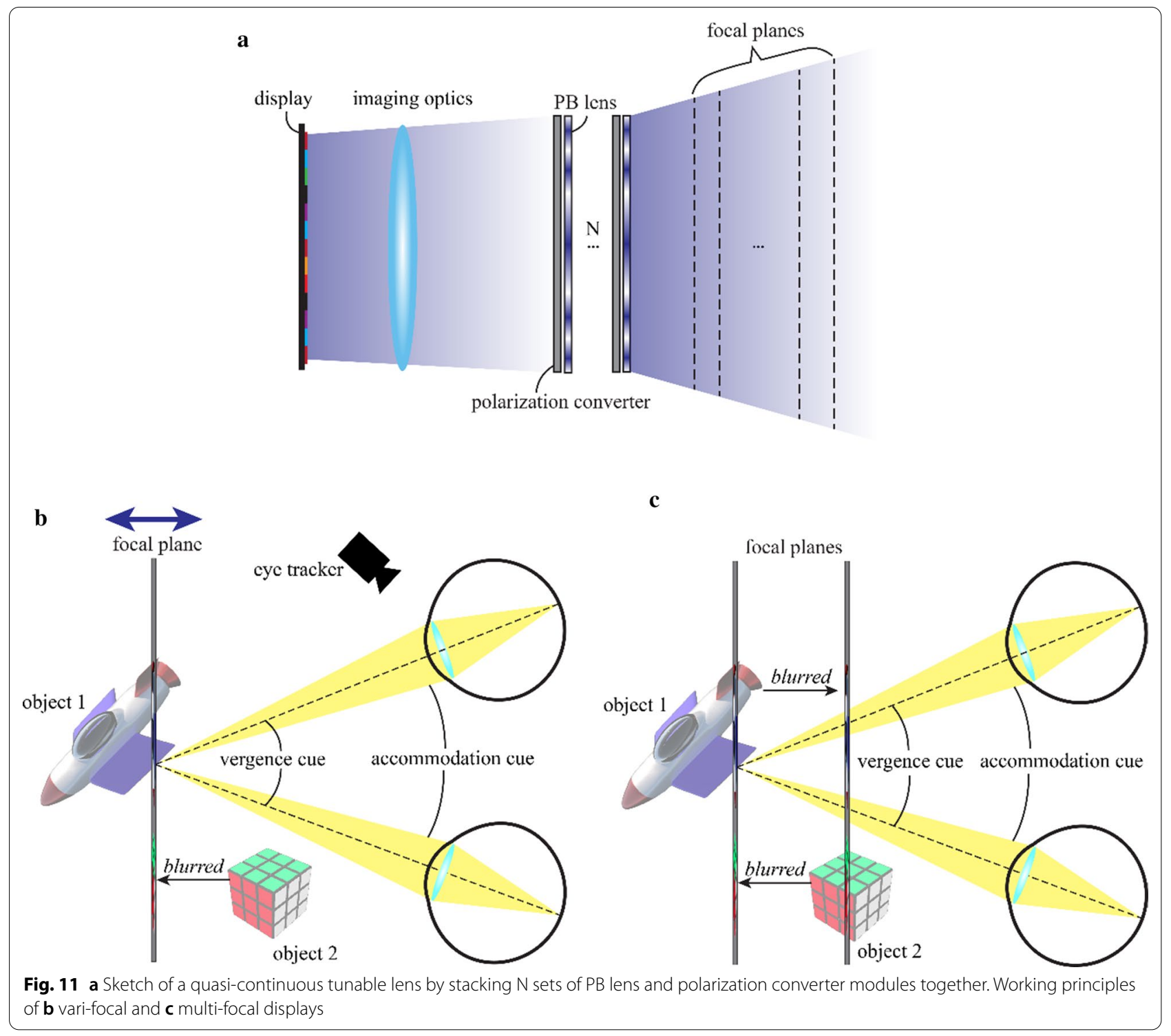

For a fixed configuration, the PB lens can be used to alleviate original chromatic aberration in a system [86]. For a system with multiple focal planes, the situation is more complicated. In theory, we can still optimize the chromatic aberration for a specific depth. For other depths, digital corrections may be applied to alleviate the issue.

\subsection{Augmented reality displays}

As discussed above, VR displays have a relatively simple optical system with a display panel and a collimation optics. The display panel size could be large and placed in front of the viewer's eyes. However, for AR displays the situation is very different. The requirement of see-through capability rules out the possibility of placing a display panel in front of the eye. Rather, an optical combiner that lets environment light passing through is configured at the front. The display module is usually placed on the backside of the combiner, along the eyeglass temples. Therefore, the display module should have a small size to fit into the compact, eyeglass-like form factor. A small display module, however, brings up the étendue problem. In a traditional geometric optical system with refractive and reflective surfaces, the étendue of system is conserved, which can be expressed as

$$
\text { Étendue }=D_{\text {display }} \cdot N A=D_{E P} \cdot \sin \left(\frac{F o V}{2}\right)
$$

where $D_{\text {display }}$ is the size of display panel, $N A$ is the system's numerical aperture, $D_{E P}$ is the size of exit pupil, and FoV is the field of view. There is a clear trade-off between 
FoV and exit pupil size, both of which are desired in an AR system. To increase both FoV and exit pupil size means to increase the system étendue, which encounters design difficulty mentioned above.

To avoid this issue, waveguide display is proposed [2, 100]. As shown in Fig. 12a, the emitted light from display panel is firstly collimated by a lens. The input light is diffracted by the in-coupling grating to an angle which satisfies the total internal reflection (TIR) condition. Such a propagating light then encounters the out-coupling grating multiple times and gets diffracted out of the glass substrate. Each time the light encounters the out-coupling grating, the system étendue increases. This can also be interpreted by the fact that the eyebox is increased in each out-coupling without influencing FoV. Although grating is used here as an example, the in-coupler and out-coupler can also be reflective mirrors [101, 102]. Those types of display are usually referred as "lightguide" displays.

Presently, some waveguide based commercial AR products use surface-relief gratings, which are fabricated by nanoimprint and photolithography processes. The in-coupler should have a high efficiency to collect all the light from image source. The out-coupler, on the other hand, should have a low but gradient efficiency, which can be achieved by the modulation of grating depth. The purpose is to obtain a relatively uniform light intensity distribution for the out-coupled light. Ideally, the grating efficiency should gradually increase from the in-coupler side to the out-coupler side. However, such a gradient efficiency makes the brightness of environment light non-uniform.

Reflective LC gratings can also be used in waveguide displays, with additional degrees of freedom for polarization management. As illustrated in Fig. 12b, when the out-coupler is a reflective LC grating, the grating itself is uniform except that a polarization management layer (PML) is laminated to the bottom substrate. The LC molecules inside the PML have spatially variant distributions, which provides additional design freedom for optimization. Whenever the TIR-guided light hits the PML, it experiences a polarization state change. As a result, the efficiency of next diffraction from the LC grating will change. Such a uniform LC grating with PML not only provides a method to control the uniformity of display light with potentially more degrees of freedom but also eliminates the non-uniformity of background light.

Aside from waveguide displays, there is another type of display with potential for overcoming the étendue issue, called Maxwellian display [103-105]. The basic working principle of Maxwellian display is depicted in Fig. 13a. The output light from a point source is collimated by a lens and passes a spatial light modulator (SLM), forming

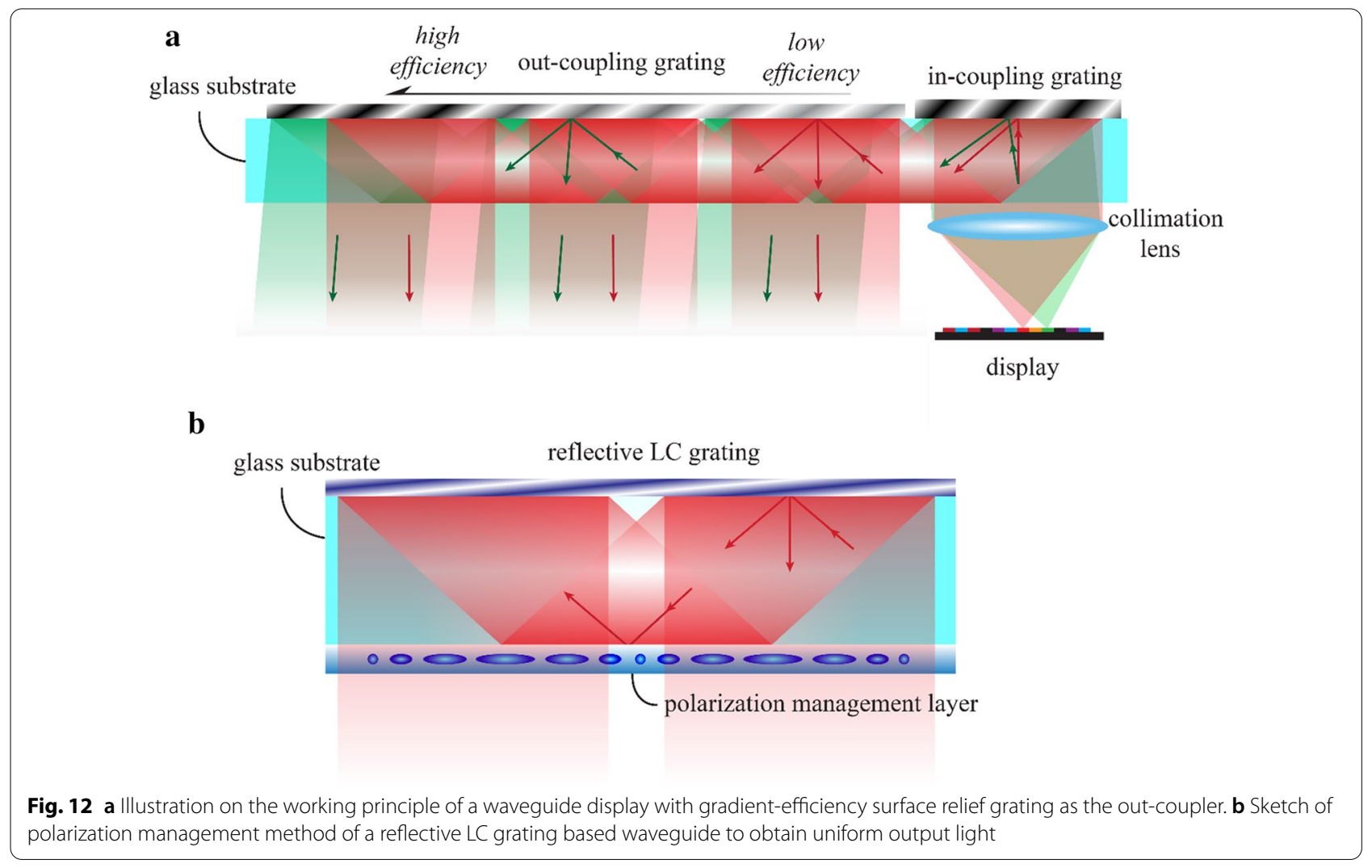




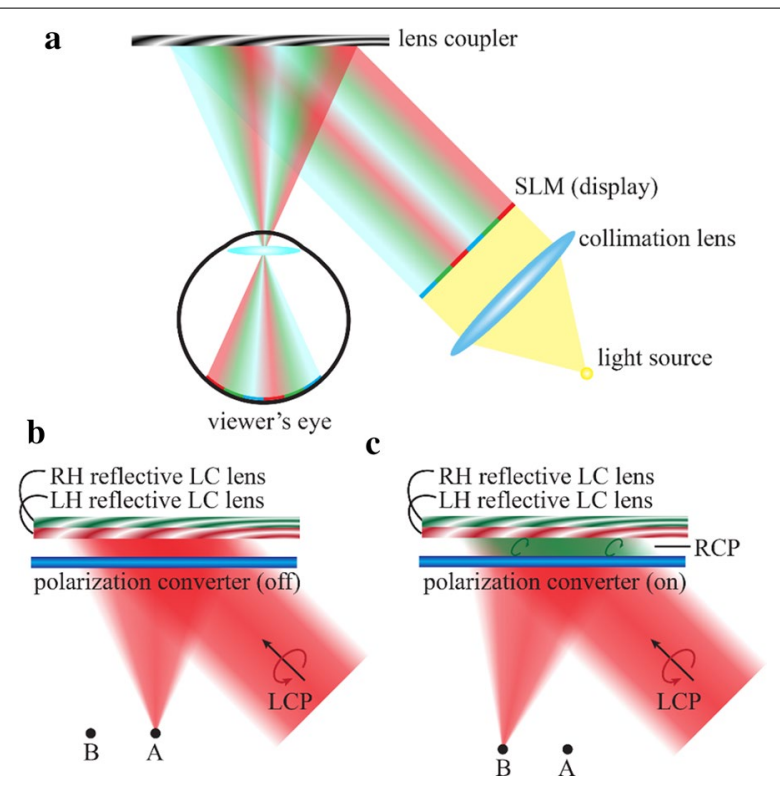

Fig. 13 a Working principle of a Maxwellian display. Pupil steering method with reflective $L C$ lenses. The light is steered to $\mathbf{b}$ viewpoint $A$ and $\mathbf{c}$ viewpoint $\mathrm{B}$

an image. The SLM can also be replaced by a laser scanning system using micro-electro-mechanical system (MEMS). The light is then focused by a lens coupler, forming a viewpoint on the viewer's eye pupil. The image is directly projected onto the retina. The viewpoint can be considered as the image of light source. If the viewpoint is significantly smaller than the eye's pupil size, then the adjustment of eye lens has no influence on the formed image on retina. Therefore, the image is always in-focus, which also partially solves the VAC issue because the accommodation cue is absent. The FoV is determined by the $f / \#$ of the lens coupler and can be increased without limit. The eyebox, however, is tiny and has the same size as the eye pupil diameter, which typically ranges from 2 to $4 \mathrm{~mm}$ in bright environment. How to enlarge the eyebox is considered the most important task for Maxwellian displays.

Using the above-described polarization-dependent LC optical elements, we can steer the viewpoint in an elegant way, without the need of mechanical components [106, 107]. As sketched in Fig. 13b, c, two reflective LC lenses with opposite handedness are used as coupler. Here, the left-handed (LH) reflective LC lens responds to the LCP light and the right-handed (RH) one to the RCP light. A polarization converter is used to switch the incident LCP and RCP states. The polarization converter can be an active LC cell with homogeneous alignment or vertical alignment for a narrow-band light source or twistednematic alignment for a broadband light source. When the polarization converter is at voltage-off state, the incident LCP light is deflected by the LH lens into viewpoint $\mathrm{A}$. When the polarization converter is activated, it switches the input LCP light into RCP light, which firstly passes through the LH lens and gets deflected by the $\mathrm{RH}$ lens into viewpoint B. Of course, the illustrated setup only serves as one of many possible cases. By stacking multiple lens-converter sets together, we can steer light into multiple viewpoints. The employed two LC lenses can also have same handedness, with a polarization converter sandwiched in between.

Waveguide displays exhibits a large eyebox, but the FoV is constrained by the TIR process. Maxwellian displays, on the other hand, has tiny eyebox, but the FoV is not limited. Lately, an interesting display system based on flat LC optics has been proposed by combining the advantages of these two approaches, called scanning waveguide display $[57,108]$. The working principle is shown in Fig. 14. The light from a laser beam scanning system is collimated by a lens and then diffracted into waveguide

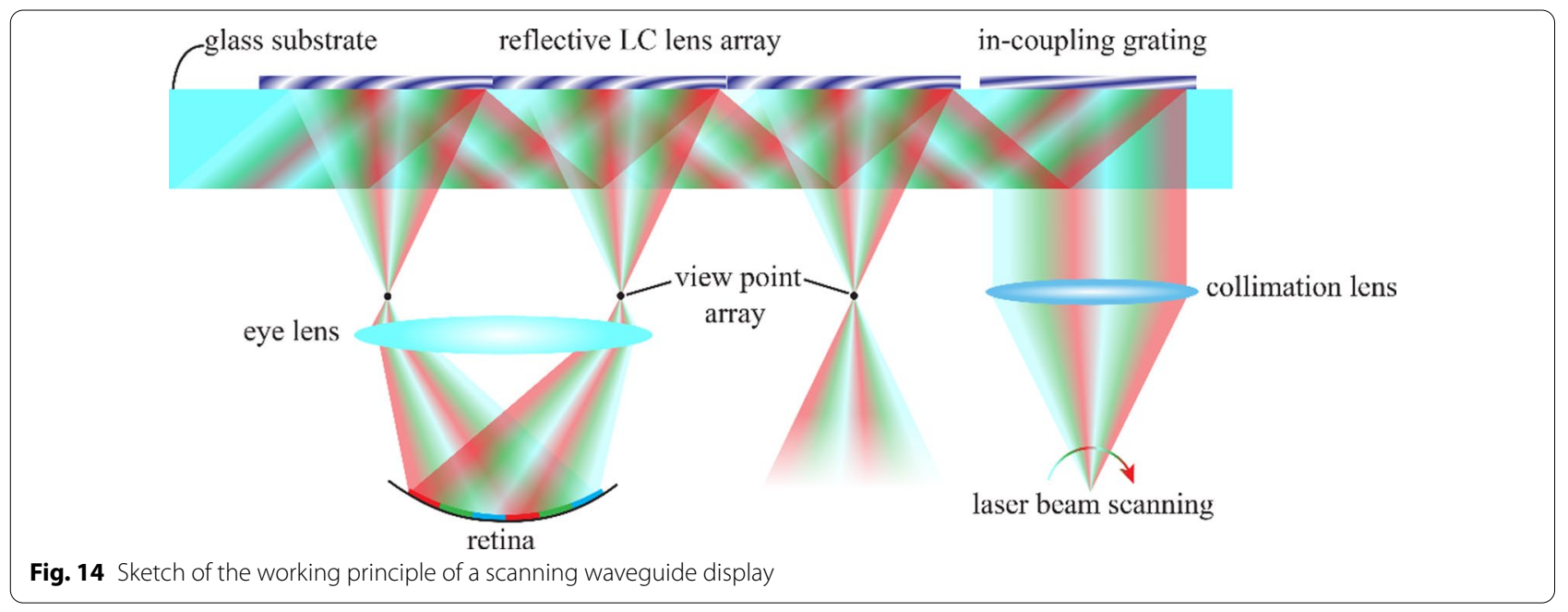


by the in-coupling grating. Instead of a grating, a LC lens array with low $f / \#$ is used as the out-coupler. As light propagates in the waveguide, it encounters the lens array and gets coupled out for multiple times. An array of viewpoints is formed. The light rays of these viewpoints are parallel to each other. Thus, these rays are converged to a single point on the viewer's retina, forming an intended image. With expanded eyebox from the TIR process, and the large FoV from the low $f / \#$ lens array, the scanning waveguide display can achieve an unprecedented large étendue, while maintaining a favorable form factor.

\section{Conclusions}

We have briefly reviewed the operation principles, fabrication processes, and potential applications of diffractive LC optical elements. Some major problems in current VR and AR systems, such as VAC, FoV, and form factor can be mitigated by the unique optical properties of these LC devices. Advantages of these planar LC optical elements like high efficiency, compact form, dynamic switching ability, polarization-dependent behaviors, and high degrees of design freedom are expected to continue impacting next-generation imaging and display systems.

\section{Acknowledgements}

The authors are indebted to Tao Zhan, Kun Yin, and Dr. Kun Li for useful discussion.

\section{Authors' contributions}

JX prepared the manuscript. JX and S-TW edited the manuscript. Both authors read and approved the final manuscript.

\section{Funding}

Goertek Electronics.

\section{Availability of data and materials}

All data and materials needed to evaluate the conclusions in the paper are present in the paper. Additional data related to this paper may be requested from the authors.

\section{Declarations}

\section{Competing interests}

The authors declare that they have no competing interests.

Received: 15 February 2021 Revised: 13 April 2021 Accepted: 27 April 2021

Published online: 08 June 2021

\section{References}

1. T. Zhan, K. Yin, J. Xiong, Z. He, S.-T. Wu, Augmented reality and virtual reality displays: perspectives and challenges. iScience 23, 101397 (2020)

2. Y.-H. Lee, T. Zhan, S.-T. Wu, Prospects and challenges in augmented reality displays. Virtual Reality Intell. Hardware 1, 10 (2019)

3. Z. Feng, H. B. Duh, M. Billinghurst, in 2008 7th IEEE/ACM International Symposium on Mixed and Augmented Reality2008), pp. 193.

4. O. Cakmakci, J. Rolland, Head-worn displays: a review. J. Disp. Technol. 2 199 (2006)
5. B. Wheelwright, Y. Sulai, Y. Geng, S. Luanava, S. Choi, W. Gao, J. Gollier, in Digital Optics for Immersive Displays (International Society for Optics and Photonics, 2018), pp. 1067604

6. G. Tan, Y. Huang, M.-C. Li, S.-L. Lee, S.-T. Wu, High dynamic range liquid crystal displays with a mini-LED backlight. Opt. Express 26, 16572 (2018)

7. H. Seetzen, W. Heidrich, W. Stuerzlinger, G. Ward, L. Whitehead, M. Trentacoste, A. Ghosh, A. Vorozcovs, in ACM SIGGRAPH 2004 Papers (Association for Computing Machinery, Los Angeles, California, 2004), pp. 760.

8. C.A. Curcio, K.R. Sloan, R.E. Kalina, A.E. Hendrickson, Human photoreceptor topography. J. Comp. Neurol. 292, 497 (1990)

9. G. Kramida, Resolving the vergence-accommodation conflict in headmounted displays. IEEE Trans. Vis. Comput. Graph. 22, 1912 (2016)

10. S. Reichelt, R. Häussler, G. Fütterer, N. Leister, in Three-Dimensional Imaging, Visualization, and Display 2010 and Display Technologies and Applications for Defense, Security, and Avionics IV (International Society for Optics and Photonics, 2010), pp. 76900B.

11. Y. Huang, E.-L. Hsiang, M.-Y. Deng, S.-T. Wu, Mini-LED, micro-LED and OLED displays: present status and future perspectives. Light Sci. Appl. 9, 105 (2020)

12. H.-W. Chen, J.-H. Lee, B.-Y. Lin, S. Chen, S.-T. Wu, Liquid crystal display and organic light-emitting diode display: present status and future perspectives. Light Sci. Appl. 7, 17168 (2018)

13. K. Yin, J. Xiong, Z. He, S.T. Wu, Patterning liquid-crystal alignment for ultrathin flat optics. ACS Omega 5, 31485 (2020)

14. J. Xiong, K. Yin, K. Li, S.-T. Wu, Holographic optical elements for augmented reality: principles, present status, and future perspectives. Adv. Photonics Res. 2, 2000049 (2021)

15. N.V. Tabiryan, S.V. Serak, D.E. Roberts, D.M. Steeves, B.R. Kimball, Thin waveplate lenses of switchable focal length—new generation in optics. Opt. Express 23, 25783 (2015)

16. N.V. Tabiryan et al., Advances in transparent planar optics: enabling large aperture. Ultrathin Lenses. Adv. Opt. Mater. 9, 2001692 (2021)

17. P.-G. De. Gennes, J. Prost, The Physics of Liquid Crystals (Oxford University Press, Oxford, 1993)

18. Y. Chen, S.-T. Wu, Recent advances on polymer-stabilized blue phase liquid crystal materials and devices. J. Appl. Polym. Sci. 131, 40556 (2014)

19. W. Zhao, C.-X. Wu, M. Iwamoto, Analysis of weak-anchoring effect in nematic liquid crystals. Phys. Rev. E 62, R1481 (2000)

20. S.V. Shiyanovskii, A. Glushchenko, Y. Reznikov, O.D. Lavrentovich, J.L. West, Tensor and complex anchoring in liquid crystals. Phys. Rev. E 62, R1477 (2000)

21. Y.-H. Lin, Y.-J. Wang, V. Reshetnyak, Liquid crystal lenses with tunable focal length. Liq. Cryst. Rev. 5, 111 (2017)

22. A. Jamali, D. Bryant, Y. Zhang, A. Grunnet-Jepsen, A. Bhowmik, P.J. Bos, Design of a large aperture tunable refractive Fresnel liquid crystal lens. Appl. Opt. 57, B10 (2018)

23. C. Yousefzadeh, A. Van Rynbach, P.J. Bos, Design of a large aperture, tunable, Pancharatnam phase beam steering device. Opt. Express 28, 991 (2020)

24. D. Xu, G. Tan, S.T. Wu, Large-angle and high-efficiency tunable phase grating using fringe field switching liquid crystal. Opt. Express 23, 12274 (2015)

25. D.P. Resler, D.S. Hobbs, R.C. Sharp, L.J. Friedman, T.A. Dorschner, Highefficiency liquid-crystal optical phased-array beam steering. Opt. Lett. 21, 689 (1996)

26. B. Apter, U. Efron, E. Bahat-Treidel, On the fringing-field effect in liquidcrystal beam-steering devices. Appl. Opt. 43, 11 (2004)

27. S. Varghese, G.P. Crawford, C.W.M. Bastiaansen, D.K.G. de Boer, D.J. Broer, Microrubbing technique to produce high pretilt multidomain liquid crystal alignment. Appl. Phys. Lett. 85, 230 (2004)

28. M. Honma, T. Nose, Twisted nematic liquid crystal polarization grating with the handedness conservation of a circularly polarized state. Opt Express 20, 18449 (2012)

29. J. Kim, J.-H. Na, S.-D. Lee, Fully continuous liquid crystal diffraction grating with alternating semi-circular alignment by imprinting. Opt. Express 20, 3034 (2012)

30. Z. He, Y.H. Lee, R. Chen, D. Chanda, S.-T. Wu, Switchable PancharatnamBerry microlens array with nano-imprinted liquid crystal alignment. Opt. Lett. 43, 5062 (2018)

31. O. Yaroshchuk, Y. Reznikov, Photoalignment of liquid crystals: basics and current trends. J. Mater. Chem. 22, 286 (2012) 
32. K. Takatoh, M. Sakamoto, R. Hasegawa, M. Koden, N. Itoh, M. Hasegawa, Alignment Technology and Applications of Liquid Crystal Devices (CRC Press, Boca Raton, 2005)

33. H. Seiberle, M. Schadt, Photoalignment and photo-patterning of planar and homeotropic liquid-crystal-display configurations. J. Soc. Inf. Disp. 8 67 (2000)

34. M. Schadt, H. Seiberle, A. Schuster, S.M. Kelly, Photo-generation of linearly polymerized liquid crystal aligning layers comprising novel, integrated optically patterned retarders and color filters. Jpn. J. Appl. Phys. 34, 3240 (1995)

35. M. Schadt, H. Seiberle, A. Schuster, Optical patterning of multi-domain liquid-crystal displays with wide viewing angles. Nature 381, 212 (1996)

36. V.G. Chigrinov, V.M. Kozenkov, H.-S. Kwok, Photoalignment of Liquid Crystalline Materials: Physics and Applications (Wiley, Chichester, 2008)

37. T. Todorov, L. Nikolova, N. Tomova, Polarization holography. 1: A new high-efficiency organic material with reversible photoinduced birefringence. Appl. Opt. 23, 4309 (1984)

38. H. Ono, F. Takahashi, A. Emoto, N. Kawatsuki, Polarization holograms in azo dye-doped polymer dissolved liquid crystal composites. J. Appl. Phys. 97, 053508 (2005)

39. H. Gao, J. Liu, F. Gan, B. Ma, Investigation of multiple holographic recording in azo-dye-doped nematic liquid-crystal film. Appl. Opt. 48, 3014 (2009)

40. A. Shishido, Rewritable holograms based on azobenzene-containing liquid-crystalline polymers. Polym. J. 42, 525 (2010)

41. L. Nikolova, T. Todorov, M. Ivanov, F. Andruzzi, S. Hvilsted, P.S. Ramanujam, Polarization holographic gratings in side-chain azobenzene polyesters with linear and circular photoanisotropy. Appl. Opt. 35, 3835 (1996)

42. J. Minabe, T. Maruyama, S. Yasuda, K. Kawano, K. Hayashi, Y. Ogasawara, Design of dye concentrations in azobenzene-containing polymer films for volume holographic storage. Jpn. J. Appl. Phys. 43, 4964 (2004)

43. M. Häckel, L. Kador, D. Kropp, C. Frenz, H.W. Schmidt, Holographic gratings in diblock copolymers with azobenzene and mesogenic side groups in the photoaddressable dispersed phase. Adv. Funct. Mater 15, 1722 (2005)

44. O. Yaroshchuk, A.D. Kiselev, Y. Zakrevskyy, J. Stumpe, J. Lindau, Spatial reorientation of azobenzene side groups of a liquid crystalline polymer induced by linearly polarized light. Eur. Phys. J. E 6, 57 (2001)

45. A.D. Kiselev, V.G. Chigrinov, S.V. Pasechnik, A.V. Dubtsov, Photoinduced reordering in thin azo-dye films and light-induced reorientation dynamics of the nematic liquid-crystal easy axis. Phys. Rev. E 86, 011706 (2012)

46. M. Schadt, K. Schmitt, V. Kozinkov, V. Chigrinov, Surface-induced parallel alignment of liquid crystals by linearly polymerized photopolymers. Jpn. J. Appl. Phys. 31, 2155 (1992)

47. K. Ichimura, Photoalignment of liquid-crystal systems. Chem. Rev. 100, 1847 (2000)

48. C. Provenzano, P. Pagliusi, G. Cipparrone, Electrically tunable two-dimensional liquid crystals gratings induced by polarization holography. Opt. Express 15, 5872 (2007)

49. C. Provenzano, P. Pagliusi, G. Cipparrone, Highly efficient liquid crystal based diffraction grating induced by polarization holograms at the aligning surfaces. Appl. Phys. Lett. 89, 121105 (2006)

50. G.P. Crawford, J.N. Eakin, M.D. Radcliffe, A. Callan-Jones, R.A. Pelcovits, Liquid-crystal diffraction gratings using polarization holography alignment techniques. J. Appl. Phys. 98, 123102 (2005)

51. J. Xiong, T. Zhan, S.-T. Wu, A versatile method for fabricating Pancharatnam-Berry micro-optical elements. Opt. Express 27, 27831 (2019)

52. N.V. Tabiryan, S.V. Serak, S.R. Nersisyan, D.E. Roberts, B.Y. Zeldovich, D.M. Steeves, B.R. Kimball, Broadband waveplate lenses. Opt. Express 24, 7091 (2016)

53. C. Oh, M.J. Escuti, Achromatic diffraction from polarization gratings with high efficiency. Opt. Lett. 33, 2287 (2008)

54. J. Kobashi, H. Yoshida, M. Ozaki, Planar optics with patterned chiral liquid crystals. Nat. Photonics 10, 389 (2016)

55. K. Gao, H.-H. Cheng, A.K. Bhowmik, P.J. Bos, Thin-film Pancharatnam lens with low f-number and high quality. Opt. Express 23, 26086 (2015)

56. C.-J.Yun, J.-K. Song, Functional films using reactive mesogens for display applications. J. Inf. Disp. 18, 119 (2017)

57. J. Xiong, G. Tan, T. Zhan, S.-T. Wu, Breaking the field-of-view limit in augmented reality with a scanning waveguide display. OSA Continuum 3. $2730(2020)$
58. M. Schadt, R. Buchecker, K. Müller, Invited Lecture. Material properties, structural relations with molecular ensembles and electro-optical performance of new bicyclohexane liquid crystals in field-effect liquid crystal displays. Liq. Cryst. 5, 293 (1989)

59. M. Engel, G. Bernatz, A. Götz, H. Hirschmann, S. K. Lee, in SID Symposium Digest of Technical Papers (Wiley Online Library, 2015), pp. 645.

60. H. Sarkissian, B. Park, N. Tabirian, B. Zeldovich, Periodically aligned liquid crystal: potential application for projection displays. Mol. Cryst. Liq. Cryst. 451, 1 (2006)

61. R.K. Komanduri, M.J. Escuti, Elastic continuum analysis of the liquid crystal polarization grating. Phys. Rev. E 76, 021701 (2007)

62. J. Xiong, R. Chen, S.-T. Wu, Device simulation of liquid crystal polarization gratings. Opt. Express 27, 18102 (2019)

63. Y.-H. Lee, Z. He, S.-T. Wu, Optical properties of reflective liquid crystal polarization volume gratings. J. Opt. Soc. Am. B 36, D9 (2019)

64. T. Zhan, J. Xiong, Y.-H. Lee, R. Chen, S.-T. Wu, Fabrication of PancharatnamBerry phase optical elements with highly stable polarization holography. Opt. Express 27, 2632 (2019)

65. T. Zhan et al., Pancharatnam-Berry optical elements for head-up and near-eye displays [invited]. J. Opt. Soc. Am. B 36, D52 (2019)

66. H. Wu, W. Hu, H.-C. Hu, X.-W. Lin, G. Zhu, J.-W. Choi, V. Chigrinov, Y.-Q. Lu, Arbitrary photo-patterning in liquid crystal alignments using DMD based lithography system. Opt. Express 20, 16684 (2012)

67. Y. Li, Y. Liu, S. Li, P. Zhou, T. Zhan, Q. Chen, Y. Su, S.-T. Wu, Single-exposure fabrication of tunable Pancharatnam-Berry devices using a dye-doped liquid crystal. Opt. Express 27, 9054 (2019)

68. L. De. Sio, D.E. Roberts, Z. Liao, S. Nersisyan, O. Uskova, L. Wickboldt, N. Tabiryan, D.M. Steeves, B.R. Kimball, Digital polarization holography advancing geometrical phase optics. Opt. Express 24, 18297 (2016)

69. M.N. Miskiewicz, M.J. Escuti, Direct-writing of complex liquid crystal patterns. Opt. Express 22, 12691 (2014)

70. J. Kim, Y. Li, M.N. Miskiewicz, C. Oh, M.W. Kudenov, M.J. Escuti, Fabrication of ideal geometric-phase holograms with arbitrary wavefronts. Optica $\mathbf{2}$, 958 (2015)

71. M. Fratz, S. Sinzinger, D. Giel, Design and fabrication of polarization-holographic elements for laser beam shaping. Appl. Opt. 48, 2669 (2009)

72. Y. Guo, M. Jiang, C. Peng, K. Sun, O. Yaroshchuk, O. Lavrentovich, Q.-H. Wei, High-resolution and high-throughput plasmonic photopatterning of complex molecular orientations in liquid crystals. Adv. Mater. 28, 2353 (2016)

73. M. Jiang, Y. Guo, H. Yu, Z. Zhou, T. Turiv, O.D. Lavrentovich, Q.-H. Wei, Low f-number diffraction-limited Pancharatnam-Berry microlenses enabled by plasmonic photopatterning of liquid crystal polymers. Adv. Mater. 31, 1808028 (2019)

74. S.R. Nersisyan, N.V. Tabiryan, D.M. Steeves, B.R. Kimball, Characterization of optically imprinted polarization gratings. Appl. Opt. 48, 4062 (2009)

75. T. Zhan, J. Xiong, G. Tan, S.-T. Wu, Absorption-based polarization gratings. Opt. Express 28, 13907 (2020)

76. X. Xiang, J. Kim, M.J. Escuti, Bragg polarization gratings for wide angular bandwidth and high efficiency at steep deflection angles. Sci. Rep. 8 , 7202 (2018)

77. K. Gao, C. McGinty, H. Payson, S. Berry, J. Vornehm, V. Finnemeyer, B. Roberts, P. Bos, High-efficiency large-angle Pancharatnam phase deflector based on dual-twist design. Opt. Express 25, 6283 (2017)

78. D.-K. Yang, S.-T. Wu, Fundamentals of Liquid Crystal Devices, 2nd edn. (Wiley, Chichester, 2015)

79. R. Chen, Y.-H. Lee, T. Zhan, K. Yin, Z. An, S.-T. Wu, Multistimuli-responsive self-organized liquid crystal Bragg gratings. Adv. Opt. Mater. 7, 1900101 (2019)

80. M.G. Moharam, L. Young, Criterion for Bragg and Raman-Nath diffraction regimes. Appl. Opt. 17, 1757 (1978)

81. J. Xiong, S.-T. Wu, Rigorous coupled-wave analysis of liquid crystal polarization gratings. Opt. Express 28, 35960 (2020)

82. M.G. Moharam, E.B. Grann, D.A. Pommet, T.K. Gaylord, Formulation for stable and efficient implementation of the rigorous coupled-wave analysis of binary gratings. J. Opt. Soc. Am. A 12, 1068 (1995)

83. M.G. Moharam, T.K. Gaylord, Rigorous coupled-wave analysis of planargrating diffraction. J. Opt. Soc. Am. 71, 811 (1981)

84. P. Kurzynowski, W.A. Woźniak, M. Szarycz, Geometric phase: two triangles on the Poincaré sphere. J. Opt. Soc. Am. A 28, 475 (2011) 
85. J. Zou, T. Zhan, J. Xiong, S.-T. Wu, Broadband wide-view PancharatnamBerry phase deflector. Opt. Express 28, 4921 (2020)

86. T. Zhan, J. Zou, J. Xiong, X. Liu, H. Chen, J. Yang, S. Liu, Y. Dong, S.-T. Wu, Practical chromatic aberration correction in virtual reality displays enabled by cost-effective ultra-broadband liquid crystal polymer lenses. Adv. Opt. Mater. 8, 1901360 (2020)

87. M. Schadt, W. Helfrich, Voltage-dependent optical activity of a twisted nematic liquid crystal. Appl. Phys. Lett. 18, 127 (1971)

88. J. Kim, C. Oh, S. Serati, M.J. Escuti, Wide-angle, nonmechanical beam steering with high throughput utilizing polarization gratings. Appl. Opt. 50, $2636(2011)$

89. G. Tan, Y.-H. Lee, T. Zhan, J. Yang, S. Liu, D. Zhao, S.-T. Wu, Foveated imaging for near-eye displays. Opt. Express 26, 25076 (2018)

90. A. Maimone, J. Wang, Holographie optics for thin and lightweight virtual reality. ACM Trans. Graph. 39, Article 67 (2020)

91. Y. Geng et al., in Digital Optics for Immersive Displays (International Society for Optics and Photonics, 2018), pp. 1067606.

92. T. Zhan, J. Xiong, J. Zou, S.-T. Wu, Multifocal displays: review and prospect. PhotoniX 1, 10 (2020)

93. T. Zhan, Y.-H. Lee, S.-T. Wu, High-resolution additive light field near-eye display by switchable Pancharatnam-Berry phase lenses. Opt. Express 26, 4863 (2018)

94. G. Tan, T. Zhan, Y.-H. Lee, J. Xiong, S.-T. Wu, Polarization-multiplexed multiplane display. Opt. Lett. 43, 5651 (2018)

95. Y.-H. Lee et al., Recent progress in Pancharatnam-Berry phase optical elements and the applications for virtual/augmented realities. Opt. Data Process. Storage 3, 79 (2017)

96. S. Liu, H. Hua, A systematic method for designing depth-fused multi-focal plane three-dimensional displays. Opt. Express 18, 11562 (2010)

97. R. Narain, R. A. Albert, A. Bulbul, G. J. Ward, M. S. Banks, J. F. O'Brien, Optimal presentation of imagery with focus cues on multi-plane displays. ACM Trans. Graph. 34, Article 59 (2015)
98. O. Mercier, Y. Sulai, K. Mackenzie, M. Zannoli, J. Hillis, D. Nowrouzezahrai, D. Lanman, Fast gaze-contingent optimal decompositions for multifocal displays. ACM Trans. Graph. 36, Article 237 (2017)

99. J.-H. R. Chang, B. V. K. V. Kumar, A. C. Sankaranarayanan, Towards multifocal displays with dense focal stacks. ACM Trans. Graph. 37, Article 198 (2018)

100. B.C. Kress, I. Chatterjee, Waveguide combiners for mixed reality headsets: a nanophotonics design perspective. Nanophotonics 10, 41 (2021)

101. M. Xu, H. Hua, Methods of optimizing and evaluating geometrical lightguides with microstructure mirrors for augmented reality displays. Opt. Express 27, 5523 (2019)

102. D. Cheng, Y. Wang, C. Xu, W. Song, G. Jin, Design of an ultra-thin near-eye display with geometrical waveguide and freeform optics. Opt. Express 22, 20705 (2014)

103. T. Lin, T. Zhan, J. Zou, F. Fan, S.-T. Wu, Maxwellian near-eye display with an expanded eyebox. Opt. Express 28, 38616 (2020)

104. S.-B. Kim, J.-H. Park, Optical see-through Maxwellian near-to-eye display with an enlarged eyebox. Opt. Lett. 43, 767 (2018)

105. M.K. Hedili, B. Soner, E. Ulusoy, H. Urey, Light-efficient augmented reality display with steerable eyebox. Opt. Express 27, 12572 (2019)

106. J. Xiong, Y. Li, K. Li, S.-T. Wu, Aberration-free pupil steerable Maxwellian display for augmented reality with cholesteric liquid crystal holographic lenses. Opt. Lett. 46, 1760 (2021)

107. J. Xiong, Y. Li, K. Li, S.-T.Wu, Aberration-free pupil steering Maxwellian display with wide-view broadband polarization converters. J. Soc. Inf. Disp. 29, 298 (2021)

108. J. Xiong, G. Tan, T. Zhan, S.-T. Wu, Wide-view augmented reality display with diffractive cholesteric liquid crystal lens array. J. Soc. Inf. Disp. 28, 450 (2020) 